\title{
Effects of an Imposed Quasi-Biennial Oscillation in a Comprehensive Troposphere-Stratosphere-Mesosphere General Circulation Model
}

\author{
KEVIN HAMILTON \\ Geophysical Fluid Dynamics Laboratory/NOAA, Princeton University, Princeton, New Jersey
}

(Manuscript received 19 May 1997, in final form 29 October 1997)

ABSTRACT

\begin{abstract}
A 48-yr integration was performed using the Geophysical Fluid Dynamics Laboratory SKYHI tropospherestratosphere-mesosphere GCM with an imposed zonal momentum forcing designed to produce a quasi-biennial oscillation (QBO) in the tropical stratosphere. In response to this forcing, the model generates a QBO in the tropical circulation that includes some very realistic features, notably the asymmetry between the strength of the descending easterly and westerly shear zones, and the tendency for the initial westerly accelerations to appear quite narrowly confined to the equator. The extratropical circulation in the Northern Hemisphere (NH) winter stratosphere is affected by the tropical QBO in a manner similar to that observed. In particular, the polar vortex is generally weaker in winters in which there are easterlies in the tropical middle stratosphere. Roughly twothirds of the largest midwinter polar warmings occur when the equatorial $30-\mathrm{mb}$ winds are easterly, again in rough agreement with observations. Despite this effect, however, the total interannual variance in the zonalmean extratropical circulation in the model apparently is slightly decreased by the inclusion of the tropical QBO. The observed QBO dependence of the winter-mean stratospheric extratropical stationary wave patterns is also quite well reproduced in the model.

The QBO was also found to have a profound influence on stratospheric stationary waves at low latitudes. Near and above $10 \mathrm{mb}$ the $\mathrm{NH}$ stationary waves were found to penetrate across the equator during the westerly QBO phase, but to be restricted to latitudes poleward of $\sim 10^{\circ} \mathrm{N}$ during the easterly phase. This means that the equatorial $\mathrm{QBO}$ in prevailing wind near and above $10 \mathrm{mb}$ has a significant zonally asymmetric component. If this is also true in the real atmosphere, there are important implications for the adequacy of the current observational rawindsonde network near the equator.

Analysis of the zonal-mean zonal momentum budget in the tropical stratosphere reveals that the resolved waves in the model are strong enough to force the realized accelerations through much of the QBO cycle. The exception appears to be the easterly acceleration phase below about $20 \mathrm{mb}$. The implications of this for the generation of a self-consistent QBO by GCMs will be considered.

The effects of the imposed QBO on the troposphere were found to be very modest. There does appear to be a statistically significant weakening (by $\sim 1 \mathrm{~m} \mathrm{~s}^{-1}$ ) of the high-latitude winter vortex in the middle and upper troposphere. Given the very high predictability of the stratospheric QBO itself, this effect could possibly be used to enhance the skill of seasonal weather forecasts. No significant QBO influence was found in the model precipitation field.
\end{abstract}

\section{Introduction}

The quasi-biennial oscillation $(\mathrm{QBO})$ is one of the most prominent aspects of the atmospheric general circulation. The QBO in the tropical stratosphere is immediately apparent when any multiyear time series of wind or temperature data is examined (e.g., Veryard and Ebdon 1961). There also have been a large number of observational and modeling studies of the influence of the QBO outside of the tropical stratosphere. QBO effects in the extratropical stratosphere have been found in analysis of long data records, and some claims have been made as well for QBO influence in the troposphere.

Corresponding author address: Dr. Kevin Hamilton, GFDL/ NOAA, Princeton University, P.O. Box 308, Princeton, NJ 08542. E-mail: kph@gfdl.gov
Such manifestations of the QBO are much subtler than the tropical stratospheric wind variations themselves, but may still represent a significant contribution to the interannual variability in the circulation.

The clearest and best studied of these remote effects is the influence of the tropical QBO on the Northern Hemisphere $(\mathrm{NH})$ winter high-latitude stratosphere. Holton and Tan $(1980,1982)$ examined $16 \mathrm{yr}$ of $\mathrm{NH}$ geopotential height data and showed that the monthly mean strength of the polar vortex up to at least the 10$\mathrm{mb}$ level was positively correlated with the equatorial zonal wind at $50 \mathrm{mb}$ (i.e., the vortex is generally weaker in the easterly QBO phase). This tendency is also seen in the record of occurrence of major sudden warmings, which are $\sim 2-3$ times more frequent when the equatorial wind at $50 \mathrm{mb}$ is easterly than when it is westerly (Labitzke 1982; Dunkerton et al. 1988). More recently 
Baldwin and Dunkerton (1991) examined the interannual variability of the zonal-mean circulation in the $\mathrm{NH}$ winter season as revealed in $12 \mathrm{yr}$ of National Meteorological Center analyses extending up to $1 \mathrm{mb}$. The overall tendency for the $\mathrm{NH}$ vortex to be weaker in years with equatorial easterlies at lower-stratospheric levels was shown clearly in their analysis. Baldwin and Dunkerton found that on average the polar vortex is about 8 $\mathrm{m} \mathrm{s}^{-1}$ weaker when the equatorial wind at $40 \mathrm{mb}$ is easterly than when it is westerly. This effect of the lower-stratospheric QBO phase on the NH vortex extends at least up to the 1-mb level.

There have been a number of theoretical studies of the extratropical influence of the tropical mean wind variations. Plumb (1982) considered the response of a zonally symmetric model to the imposition of low-latitude zonal momentum sources. He found that the effect of the low-latitude forcing was largely confined equatorward of $\sim 35^{\circ}$, and so concluded that the purely zonally symmetric circulation could not account for the observations of Holton and Tan concerning the QBO effects on the NH polar vortex. This led to the application of models, which explicitly include at least largescale eddy motions. Some of these models have been single-layer idealizations of the atmosphere (O'Sullivan and Salby 1990; Polvani et al. 1995), whereas others have been based on the full three-dimensional primitive equations (Holton and Austin 1991; O'Sullivan and Young 1992; O'Sullivan and Dunkerton 1994; O'Sullivan and Chen 1996; Hess and O'Sullivan 1995; Chen 1996). It should be noted that all these papers report on integrations of models with simplified imposed forcings for the large-scale eddy motions that are crucial in transmitting the effects of the tropical QBO to higher latitudes. Such models have been successful in reproducing at least qualitatively the observed relation between the tropical winds and the extratropical vortex strength. The basic picture that emerges is that in the easterly QBO phase (but not in the westerly phase) there is a zero wind line in the NH subtropical lower stratosphere. The equatorward propagation of the quasi-stationary waves generated in the NH extratropics is largely stopped near the zero wind line in the subtropics. There is presumably some reflection of this wave activity from the vicinity of the zero wind line, and the net effect is to provide a more confined waveguide for the waves in the easterly QBO phase than in the westerly phase. This confined waveguide leads to more of the wave flux being focused into the polar vortex region and a consequently weaker vortex. Dunkerton and Baldwin (1991) and Baldwin and Dunkerton (1991) present observational evidence for the effects of the tropical QBO on the flux of wave activity in the extratropical quasi-stationary waves

While the mechanistic model studies have provided considerable insight into the tropical-extratropical interactions in the stratosphere, there is interest in seeing how well such effects are simulated in comprehensive general circulation models (GCMs) in which the wave forcing for the stratosphere is generated self-consistently. An obvious difficulty in such a project is the virtual lack of a tropical QBO in current GCMs [the only exception at present appears to be that reported in Takahashi (1996)]. Some earlier studies (Balchandran et al. 1991; Hamilton and Yuan 1992; Hamilton 1995a; Balachandran and Rind 1995) have examined various aspects of GCM behavior when the tropical stratospheric winds are forced strongly easterly or westerly in arbitrary ways. These experiments were all rather limited in length and involved forcing somewhat unrealistic wind fields in the tropical stratosphere. The present paper reports on an experiment conducted with the Geophysical Fluid Dynamics Laboratory SKYHI troposphere-stratosphere-mesosphere GCM in which the tropical stratospheric QBO was forced artificially by including an imposed zonally symmetric zonal momentum source. The forcing was designed to produce a quite realistic tropical mean wind evolution, and a very long integration (48 yr) was performed in order to enable detection of relatively weak effects.

A major focus in this project is on the effects of the imposed QBO on extratropical variability in the $\mathrm{NH}$ stratosphere. Modeling a realistic variability in the $\mathrm{NH}$ polar vortex is an important test for a GCM. Some reasonably promising results have been obtained with current GCMs (e.g., Hamilton 1995a), but the issue of QBO contributions to the interannual variability (virtually absent from the standard versions of most current GCMs) clearly needs to be examined. The ability of a GCM to simulate a realistic degree of interannual variability in the $\mathrm{NH}$ winter stratosphere is important for one of the most practical applications now envisaged for middle atmosphere GCMs, namely simulating the response of the stratosphere to increased concentrations of greenhouse gases. The overall cooling of the stratosphere expected from the increased IR opacity will make the occurrence of polar stratospheric clouds (PSC; and hence heterogeneous ozone loss) more likely. However, even with significantly higher greenhouse gas concentrations, the occurrence of a significant amount of PSC formation in a particular winter will depend on the dynamical evolution in that year (Austin et al. 1992; Austin and Butchart 1994). Thus an accurate prediction of the response of the stratosphere to enhanced greenhouse gas concentrations will require a model with a realistic representation of the interannual variability of the polar vortex.

There is also interest in examining the response to the imposed QBO of the model dynamics in the tropical stratosphere itself. In the control model the tropical stratospheric mean winds are rather steady and are in reasonable agreement with the long-term mean observations. However, the mean winds in the real atmosphere are dominated by the QBO, and so never at any instant resemble the long-term mean. Thus the tropical eddies in the control model never propagate through a 
realistic stratospheric mean flow profile. This complicates the evaluation of the eddy simulation in the model (particularly the eddy driving of mean flow accelerations). Forcing the model to undergo a QBO allows the eddies to be examined under realistic mean flow conditions and permits a calculation of how much extra momentum forcing is actually required to produce realistic mean flow accelerations. Thus this QBO experiment is a useful way to examine the eddy dynamics of the model in the tropical stratosphere (and may even help diagnose the deficiencies that prevent the control model from spontaneously producing a realistic QBO).

Another issue that can be addressed in the model experiments reported here is the adequacy of the current radiosonde observations in the equatorial stratosphere. In the last decade most studies of the observed QBO near the equator (e.g., Naujokat 1986; Pawson and Fiorino 1996) have relied on observations at the station at Singapore $\left(1.4^{\circ} \mathrm{N}, 103.9^{\circ} \mathrm{E}\right)$. At present there seems to be no other station near the equator that regularly reports winds up to the $10-\mathrm{mb}$ level, and the assumption is generally made that the Singapore zonal winds are representative of the whole equatorial region. Belmont and Dartt (1968) considered the question of zonal asymmetries in the QBO using zonal wind observations at several radiosonde stations then available. They concluded that the data did not clearly demonstrate significant longitudinal variations, but their analysis was restricted to levels below $50 \mathrm{mb}$. Quite recently some observations of midstratospheric winds by the high resolution doppler imager on the UARS satellite (e.g., Ortland et al. 1996) suggest that there may be some significant zonal asymmetries in the monthly mean wind at the equator in the westerly phase of the QBO (Ortland 1997). The issue of whether a single station can provide enough information for (at least) the low-frequency variations of equatorial wind has assumed some additional importance with the development of middle atmospheric data assimilation systems (e.g., Swinbank and O'Neill 1994). The present experiments will allow the examination of zonal inhomogeneities in the stratospheric flow in a model with realistic tropical zonal-mean winds.

The GCM experiment outlined here is also useful for studying the problematic issue of tropospheric influence of the stratospheric QBO. Some empirical studies have found evidence for a relation between quasi-biennial variability in the troposphere and the tropical stratospheric QBO (Ebdon 1975; Gray 1984; Mukherjee et al. 1985; Gray et al. 1992a,b). The statistical validity of some of these observations has been questioned (e.g., Trenberth 1980), and, of course, any such correlation could just as plausibly reflect tropospheric influence on the stratosphere [e.g., through the modulation of the flux of upward propagating equatorial waves by the largescale tropospheric circulation; see Maruyama and Tsuneoka (1988) and Geller et al. (1997)]. The present experiment, in which the imposed forcing is restricted to the stratosphere, allows for a clear determination of any tropospheric effects of stratospheric forcing. This is a subject of possible practical importance for seasonal weather forecasting, since the tropical QBO itself is clearly predictable with some skill out to periods of months or even years.

A final motivation for the present project is the generation of QBO variations in trace constituents. Near the equator the concentrations of $\mathrm{O}_{3}$ and other longlived trace species are observed to undergo oscillations very closely tied to the QBO zonal wind variations (e.g., Oltmanns and London 1982; Hasebe 1983; Bowman 1989; Zawodny and McCormick 1991; Randel and Wu 1996). There are also QBO-influenced interannual variations of tracer concentration observed in the subtropics and even high latitudes. These are connected to the tropical QBO in a complicated way that seems to involve a strong interaction between the $\mathrm{QBO}$ and the annual cycle (e.g., Hamilton 1989, 1995c; Holton 1989; Tung and Yang 1994). There has been a great deal of interest recently in understanding the nature of the constituent transport between the tropical and subtropical stratosphere, including the possible influence of the QBO (e.g., Hess and O'Sullivan 1995; Holton et al. 1995). A segment of the QBO GCM integration is being repeated with a version of the model with a sophisticated ozone chemistry code. The results will be described in a later publication, but the present paper provides the background to these chemical calculations.

This paper is organized as follows. Section 2 briefly describes the SKYHI GCM and the design of the QBO experiment. Section 3 covers the basic aspects of the zonal-mean circulation forced by the QBO momentum source. Section 4 discusses the effects of the tropical $\mathrm{QBO}$ on the extratropical stratospheric circulation in $\mathrm{NH}$ winter. Section 5 is a consideration of possible tropospheric influences of the imposed QBO. Those readers interested only in an overview of the results and an indication of how the addition of the QBO affects the overall performance of the model can stop at this point. Subsequent sections deal with some more detailed aspects of the analysis of the simulation. Section 6 examines the zonal-mean zonal momentum balance in the tropical stratosphere region and looks at the effects of the imposed mean flow changes on the vertically propagating waves in the model. Section 7 considers the stationary wave response in the Tropics and subtropics. The conclusions are summarized in section 8 .

\section{Model and experimental design}

This study uses the version of the SKYHI model with a $3^{\circ}$ lat by $3.6^{\circ}$ long grid and 40 levels from the ground to $0.0096 \mathrm{mb}$, as described in Hamilton et al. (1995). Aspects of the long-term mean climatology and interannual variability of this model based on a 25 -yr control run have been discussed in Hamilton et al. (1995) and Hamilton (1995a,b). Except for the addition to the zonal momentum equation described below, all aspects of the 
model formulation were kept as in Hamilton et al. (1995). This means that the model had a full annual cycle but no diurnal cycle of radiation. The sea surface temperatures and ozone concentrations were prescribed and included no interannual variation.

After the long QBO experiment described here was completed, an error was discovered that affected this experiment and the $3^{\circ} \times 3.6^{\circ}$ control run described in Hamilton et al. (but not the $1^{\circ} \times 1.2^{\circ}$ control run reported in that paper). The Fourier filtering of the highlatitude surface pressure was performed in a way that was inconsistent with the filtering of the other fields. This leads to generation of spurious eddies with short zonal scales. Ten years of the control run have now been repeated with this problem corrected. The filtering error does have some influence on the middle atmospheric climate, notably in the extratropical mesosphere. It appears that the error generates a spurious contribution to the resolved gravity wave field in the model. The gravity waves are known to be associated with a considerable wave driving of the mesospheric mean flow (e.g., Hamilton et al. 1995). Without the spurious contribution to the gravity waves, the December-February polar climatological temperatures are colder by as much as $7^{\circ} \mathrm{C}$ near $0.1 \mathrm{mb}$. The difference in zonal-mean winter climatological temperatures is mostly less than $2^{\circ} \mathrm{C}$ in the stratosphere, but rises to $3^{\circ}-4^{\circ} \mathrm{C}$ in a small region poleward of $75^{\circ} \mathrm{N}$ and between about $10 \mathrm{mb}$ and $3 \mathrm{mb}$. Effects are more dramatic in Southern Hemisphere (SH) winter, where the mesospheric polar climatological temperatures are as much as $18^{\circ}$ colder when the filtering problem is corrected. As noted by Garcia and Boville (1994), the SH winter mesosphere is the place where the model simulation is expected to be most sensitive to any augmentation of the gravity wave drag. Below $10 \mathrm{mb}$, however, differences between control and corrected June-August in the SH winter are less than $3^{\circ} \mathrm{C}$. Correcting the filtering problem leads to warmer temperatures in the summer polar mesosphere, again consistent with the inference that the filtering error produced a spurious contribution to the resolved gravity wave drag. However, the actual differences in seasonalmean, zonal-mean temperatures are smaller (less than $2^{\circ} \mathrm{C}$ ) in the summer mesosphere than in winter.

The goal of the QBO forcing was to drive the zonalmean winds in the tropical stratosphere through a very realistic QBO cycle, while leaving the extratropical circulation as unconstrained as possible. The strength of the imposed constraint was determined by trial and error. The form chosen dropped off gradually with distance from the equator in order to avoid significant discontinuities in the mean flow between the strongly constrained equatorial region and higher latitudes. The additional source in the zonal momentum was imposed very simply at each grid point

$$
\frac{\partial u}{\partial t}=\cdots-\alpha\left(\bar{u}-\bar{u}_{0}\right),
$$

where $u$ is the local zonal wind at any point; $\bar{u}$ is the zonal mean of the zonal wind; $\alpha$ is a relaxation rate, which is a prescribed function of latitude and pressure; and $\bar{u}_{0}$ is a prescribed field depending on latitude, pressure, time of year, and phase of the QBO cycle. Figure 1 shows the distribution of $\alpha$. The relaxation is quite strong (as short as a 5-day timescale) right on the equator in the middle stratosphere. The relaxation rate drops off rapidly in latitude at all heights, is zero below 103 $\mathrm{mb}$, and drops off gradually with height above $12 \mathrm{mb}$. The $\bar{u}_{0}$ field was the sum of two parts:

$$
\bar{u}_{0}=\bar{u}_{\text {clim }}+\bar{u}_{\mathrm{qbo}},
$$

where $\bar{u}_{\text {clim }}$ depends on time of year and is just the climatological mean of the control run, and

$$
\bar{u}_{\mathrm{qbo}}=U(p, t) \exp \left[-(\theta / \delta)^{2}\right],
$$

where $\theta$ is the latitude and $\delta$ is $15^{\circ}$. The time-height structure, $U$, is described in detail in appendix A. It is periodic with period $2.25 \mathrm{yr}(\sim 27$ months $)$, but involves the first six harmonics of the 27 month cycle to produce a time evolution that is closer to a square wave than a sinusoid. Figure 2 shows $\bar{u}_{\mathrm{qbo}}$ at two phases in the QBO cycle, separated by a quarter cycle. The form for $\bar{u}_{\mathrm{qbo}}$ makes no distinction between easterly and westerly phases, so the $\bar{u}_{\text {qbo }}$ profile for a half-cycle (13.5 months) later is just obtained by multiplying by -1 .

The inclusion of $\bar{u}_{\text {clim }}$ in $\bar{u}_{0}$ and the adoption of a significant relaxation rate even in the tropical mesosphere was guided by the earlier experience of Hamilton (1995a), who found that forcing the mean flow only in the tropical stratosphere resulted in the generation of unrealistically strong changes in tropical mesospheric zonal-mean wind. Since a key focus of this paper is on the extratropical response to realistic tropical QBO wind variations, it was felt advisable to constrain the tropical mesospheric winds fairly strongly as well.

The experiment began with 1 January initial conditions taken from the control run (this is year 15 in the numbering system adopted in several of the figures in this paper). The model with the momentum source was then was integrated for $48 \mathrm{yr}$. The first 35 month period of this experiment is regarded as spin-up and analysis is restricted to essentially the last $45 \mathrm{yr}$ (December of year 17 through the end of year 62). Over most of the integration, monthly means of all the full three-dimensional model variables were saved, together with daily snapshots of a large number of zonally averaged fields (including quadratic quantities such as the eddy heat and momentum transports). Additionally, during years 27-34 snapshots of the full three-dimensional wind and temperature fields were saved at 3-hourly intervals.

\section{Basic results for the low-latitude QBO}

The equatorial zonal-mean zonal wind at the $36.7-\mathrm{mb}$ model level is shown for $9 \mathrm{yr}$ of the experiment in Fig. $3 a$. The wind evolution differs significantly among the 


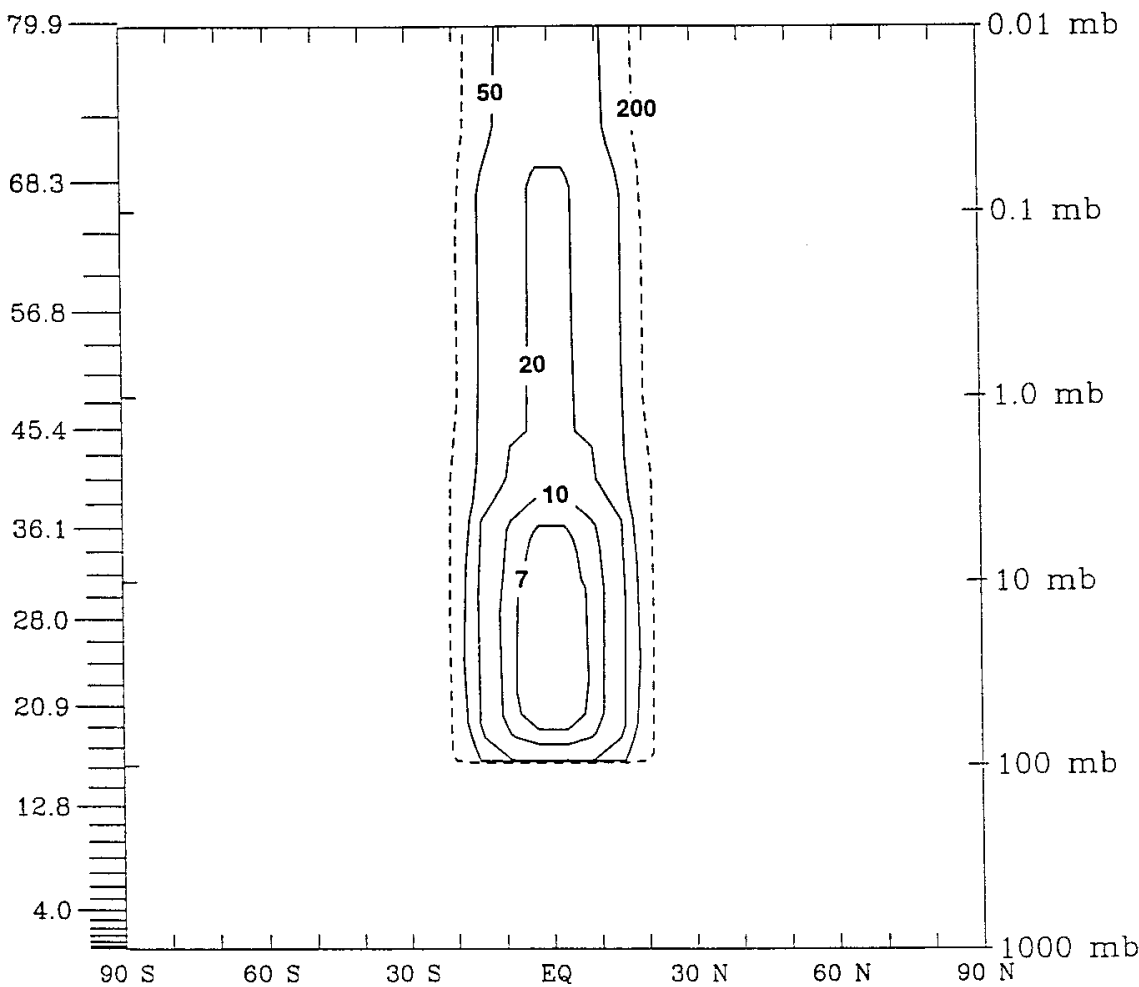

FIG. 1. The relaxation timescale $\left(\alpha^{-1}\right)$ for the imposed momentum source plotted as a function of latitude and pressure. Contours are at 7, 10, 20, 50, and 200 (dashed) days. On this and on subsequent height-latitude sections in the same format, the tick marks on the left are placed at the model level pressures. The values on the left are heights $(\mathrm{km})$ above sea level appropriate for a standard midlatitude temperature profile.

four QBO cycles within each 9-yr segment because of the relative phase of the QBO and annual cycle shifts by three months for successive cycles. The equatorial wind evolution in every fifth cycle is virtually identical. When the time series in Fig. 3a is deseasonalized it displays a very regular QBO from cycle to cycle. Figure 4a shows the height-time evolution for the equatorial zonal-mean zonal wind over a 5-yr period (corresponding to the period of the integration for which high-frequency saving is available). The QBO appears with the downward phase propagation and strong shear zones familiar from observations. Interestingly, the observed asymmetry in the strength of the shear zones [westerly shears stronger than easterly shears (e.g., Naujokat 1986)] is reproduced in the simulation, although there is no east-west asymmetry in the $\bar{u}_{\text {qbo }}$ field. Above $\sim 5$ $\mathrm{mb}$ the evolution is dominated by the semiannual oscillation (SAO), which is generated in the control model as well (Hamilton et al. 1995). Although the mean wind near the stratopause is constrained fairly strongly to relax to the climatological seasonal cycle, some substantial interannual variability does appear. Notably the westerly phase of the SAO near $1 \mathrm{mb}$ is somewhat stronger when there are easterlies throughout the lower stratosphere (in April of year 27 and April of year 29).
This QBO modulation of the stratopause SAO has some observational counterpart (Burrage et al. 1996; Garcia et al. 1997), and is the expected result of filtering of upward propagating waves by the mean flow. Figure $5 \mathrm{~b}$ shows the height-time evolution of the equatorial zonal wind when the mean seasonal cycle is removed. The very regular $\mathrm{QBO}$ up to $\sim 5 \mathrm{mb}$ (including the asymmetry between easterly and westerly shears) is evident in this figure. Near $1 \mathrm{mb}$ the deviations from the mean seasonal cycle can exceed $7.5 \mathrm{~m} \mathrm{~s}^{-1}$ on occasion, despite the constraint imposed by the mean flow relaxation.

Figure $3 \mathrm{~b}$ shows the zonal-mean equatorial temperature at $36.7 \mathrm{mb}$ for $9 \mathrm{yr}$. This raw field is somewhat noisier than the corresponding zonal wind variations, but the QBO appears quite clearly once the mean seasonal cycle is removed (not shown). This mean seasonal march of temperature is clearly dominated by an annual cycle with peak in August. Yulaeva et al. (1994) have recently examined the seasonal march of temperature in the tropical lower stratosphere and also find a strong annual cycle, which they note must be related to the differing strengths of eddy forcing of the mean flow in the NH and SH. When analyzed in a similar manner, the SKYHI mean seasonal cycle in the tropical lower 

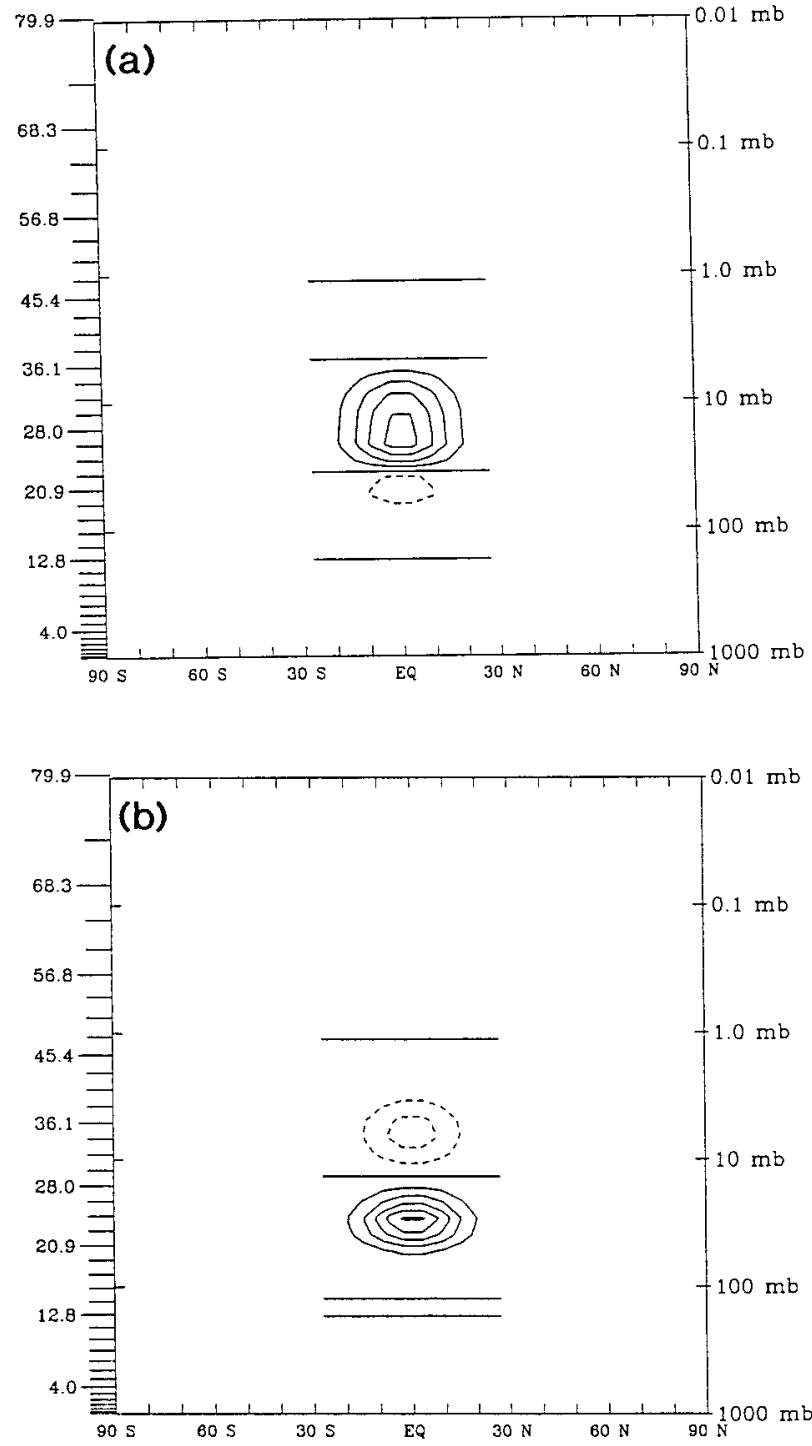

FIG. 2. The idealized QBO zonal wind distribution at two instants separated by one quarter-cycle (6.75 months). The contour interval is $5 \mathrm{~m} \mathrm{~s}^{-1}$ and dashed contours denote easterly winds. Note that the zero contours appear as horizontal lines.

stratosphere agrees very well with the observational study of Yulaeva et al. (1994).

The $\sim \pm 2{ }^{\circ} \mathrm{C}$ amplitude of the equatorial temperature QBO seen in Fig. 3 is comparable to observational estimates (e.g., Reed 1964). A direct comparison of model results with actual station observations is given in Fig. 5. The top panel shows the 12-month running mean time series of 50-mb temperature from radiosondes at Canton Island $\left(2.8^{\circ} \mathrm{S}\right)$ published by Veryard and Ebdon (1961), and the bottom shows the 12-month running mean equatorial zonal-mean temperature at the 47.9-mb model level for $6 \mathrm{yr}$ of the QBO integration. It seems that the simulation has captured the observed QBO temperature variations quite well. Note that the mean temperature in the model at this height is $\sim 5^{\circ}$ colder than in observations, consistent with earlier published comparisons (Hamilton et al. 1995).

Reed (1964) analyzed radiosonde data from about 20 stations equatorward of $25^{\circ}$ lat to determine the heightlatitude structure of the QBO. It is doubtful if enough station data are being collected today to perform an equally detailed analysis with modern data. For each station and each level, Reed performed a least-squares fit of a 26-month Fourier harmonic to the data, and used the amplitude and phase obtained as the basis for his height-latitude analysis. Figure 6 shows results of a comparable analysis of the model simulation, with the amplitude of the QBO in zonal wind shown in Fig. 6a and in temperature in Fig. 6b. These results are very close to those of Reed (1964), although the peak amplitudes in the model are slightly $(\sim 10 \%)$ larger than indicated by Reed. This difference is to be expected, since the model QBO is perfectly periodic whereas Reed fit his single harmonic to the real QBO, which, of course, has a somewhat variable period. The zonal wind amplitudes in Fig. 6a also compare well with the observational determination of Belmont et al. (1974).

Reed also produced plots showing his best estimate of the evolution of the zonal wind through a composite QBO cycle at various heights and latitudes. Figure 7 reproduces his results for $30 \mathrm{mb}$ at the equator and at $20^{\circ} \mathrm{N}$. These are compared with the same quantities from the model simulation at $28 \mathrm{mb}$ composited over the 27 month QBO cycle. Again there is very good agreement between the model and observations.

Figure 8 shows the evolution of the $28-\mathrm{mb}$ zonalmean wind in the Tropics during an easterly acceleration phase (top) and a westerly acceleration phase (bottom). Examination of these raw fields shows that the procedure adopted has succeeded in forcing the QBO without causing any significant discontinuities in the wind field between the strongly constrained equatorial region and higher latitudes. The evolution in Fig. 8 is also noteworthy for the asymmetry between westerly and easterly accelerations. Just as in observations (Hamilton 1984, 1985; Dunkerton and Delisi 1985), the initial westerly accelerations appear more narrowly confined near the equator than the initial easterly accelerations, although this asymmetry is perhaps more pronounced in the observations.

In summary, the relaxation procedure described in section 2 allowed the model to produce a rather realistic low-latitude QBO in the zonally averaged wind and temperature. Somewhat remarkably, the model simulation displays asymmetries between the easterly and westerly acceleration phases that are significant and rather realistic, despite the fact that the imposed relaxation has no explicit east-west asymmetry. Of course, there are still some somewhat unrealistic aspects of the tropical mean flow evolution. For example, the exact 27-month period assumed equally samples four different relative phases between the QBO and annual cycle, whereas 

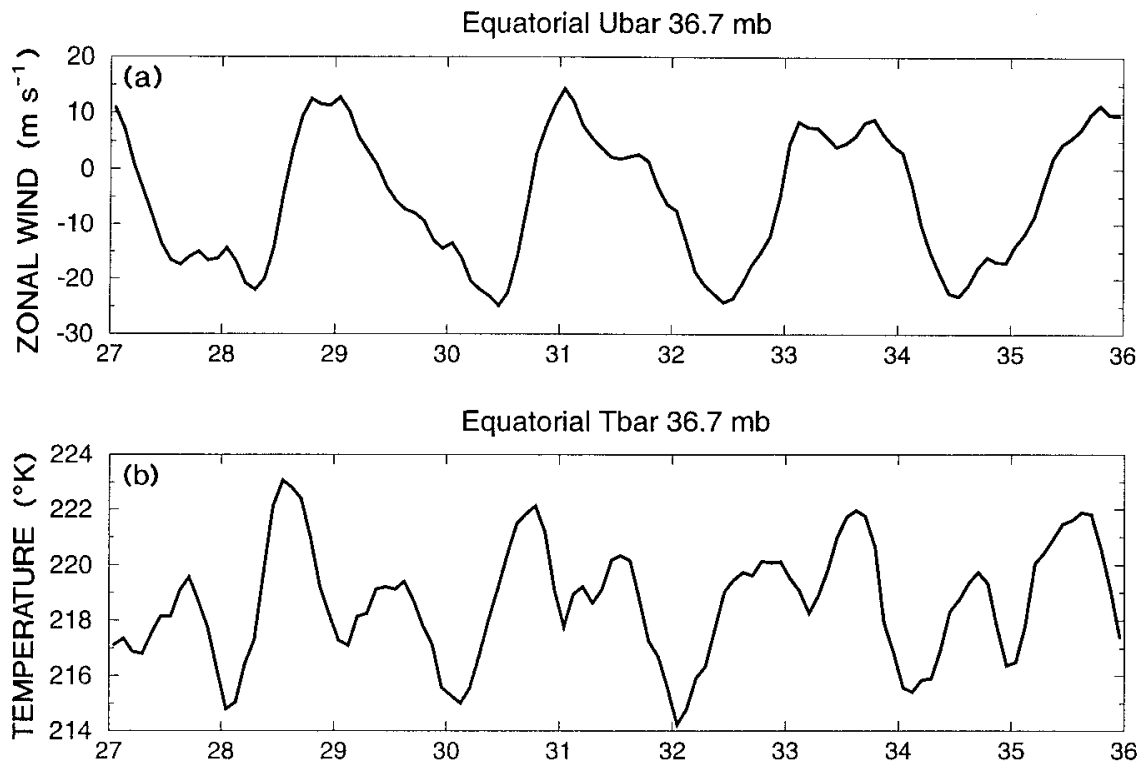

FIG. 3. (a) Time series of the monthly averaged, zonal-mean equatorial zonal wind at $36.7 \mathrm{mb}$ for $9 \mathrm{yr}$ of the QBO experiment. (b) As in panel (a) but for the temperature.

there is observational evidence for some modest preferential phase locking of the dynamical QBO with the annual cycle (e.g., Hamilton 1989).
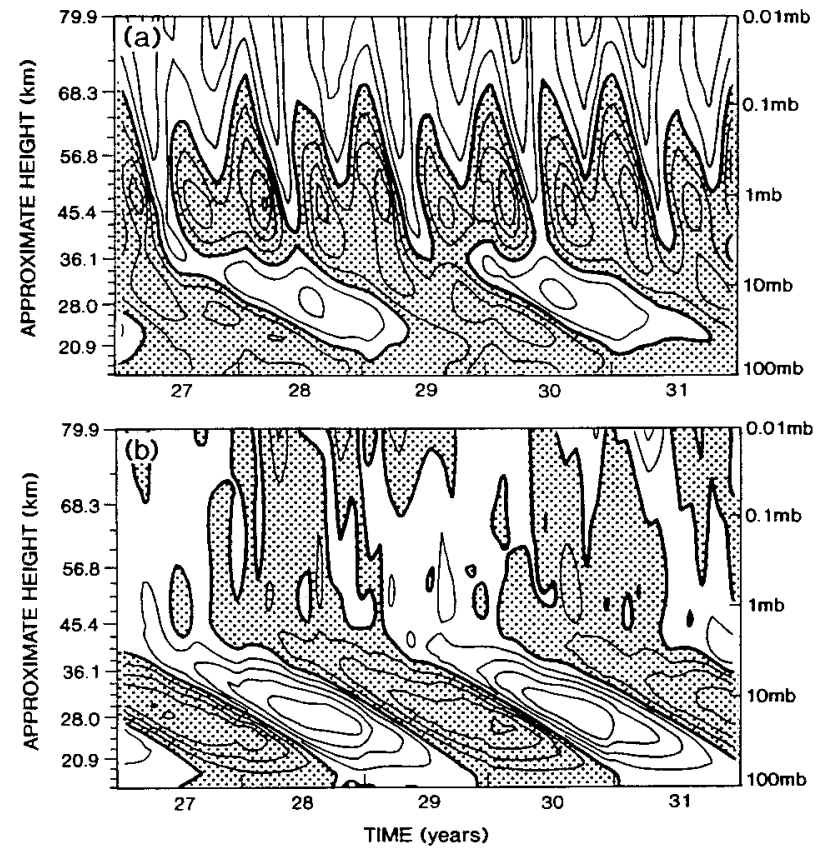

FIG. 4. (a) Time-height section of the simulated zonally averaged zonal wind at the equator for $5 \mathrm{yr}$ of the QBO experiment. Contour interval is $10 \mathrm{~m} \mathrm{~s}^{-1}$ and shading denotes easterly wind. (b) As in panel (a) but with the climatological annual cycle removed. The contour interval is $7.5 \mathrm{~m} \mathrm{~s}^{-1}$.

\section{Basic results for the extratropical middle atmospheric flow}

This section will describe the results for the QBO influence on seasonal-mean extratropical circulation in the middle atmosphere. The analysis presented here has been largely guided by published observational studies of the global effects of the QBO. Such studies have generally used the near-equatorial wind at (or near) 40 $\mathrm{mb}$ as the indicator of QBO phase. Dunkerton and Baldwin (1991) found that using the 40-mb equatorial winds produced the largest tropical-extratropical correlations, at least in $\mathrm{NH}$ winter. This is also apparent in the present modeling studies, with the largest extratropical correlations appearing with equatorial winds at either the

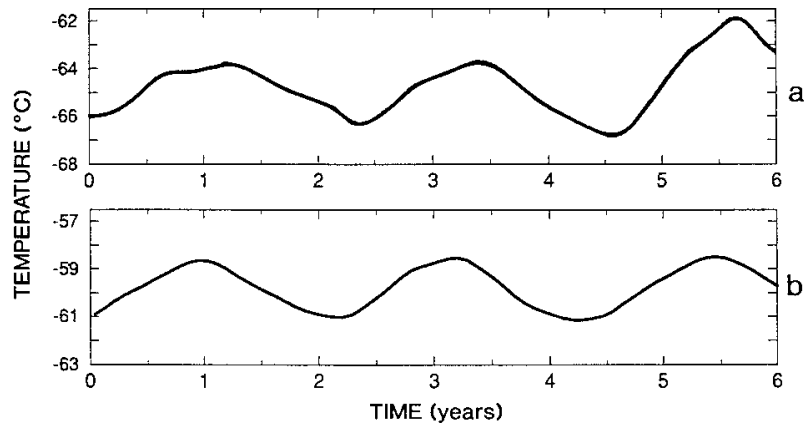

FIG. 5. (a) Twelve-month running mean of 50-mb temperature at Canton Island $\left(2.8^{\circ} \mathrm{S}, 172^{\circ} \mathrm{W}\right)$ for six consecutive years (using data from 1954 through 1960). Reproduced from Veryard and Ebdon (1961). (b) Twelve-month running mean of zonal-mean equatorial temperature at $47.9 \mathrm{mb}$ for six consecutive years of the SKYHI integration. 

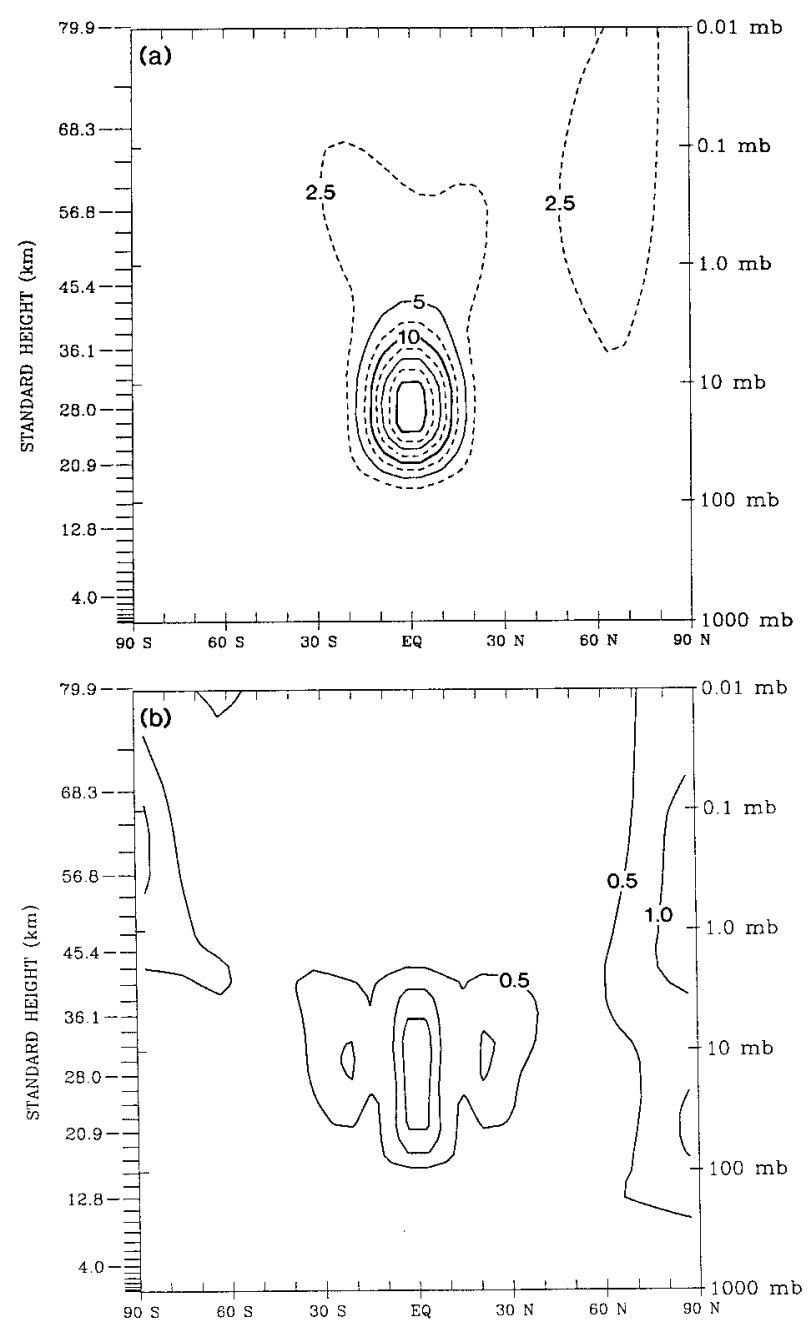

FIG. 6. (a) Amplitude of the 27-month harmonic of the zonal-mean zonal wind. Contour interval is $2.5 \mathrm{~m} \mathrm{~s}^{-1}$. Solid contours at 5,10 , 15 , and $20 \mathrm{~m} \mathrm{~s}^{-1}$, dashed contours at intermediate values. (b) Amplitude of the 27 -month harmonic of the zonal-mean temperature. Contour interval is $0.5^{\circ} \mathrm{C}$.

$36.7-\mathrm{mb}$ or $47.9-\mathrm{mb}$ model levels. In order to be consistent with published observations, the correlations and composites presented here have been based on 40-mb equatorial winds (i.e., a weighted average of the 36.7and 47.9-mb model levels). The focus here will also be on the circulation changes in $\mathrm{NH}$ winter. While there is some indication in the observed record of QBO effects in the SH winter (e.g., Garcia and Solomon 1987), these are less well documented than those in $\mathrm{NH}$ winter (e.g., Holton and Tan 1980, 1982; Baldwin and Dunkerton 1991). The SKYHI model simulation in SH winter also has some serious deficiencies (Hamilton et al. 1995; Hamilton 1995b), which make the interpretation of results there problematic. In particular, the $3^{\circ} \times 3.6^{\circ}$ resolution SKYHI simulation has a SH vortex that is much too strong and a $\mathrm{SH}$ polar region that is as much as

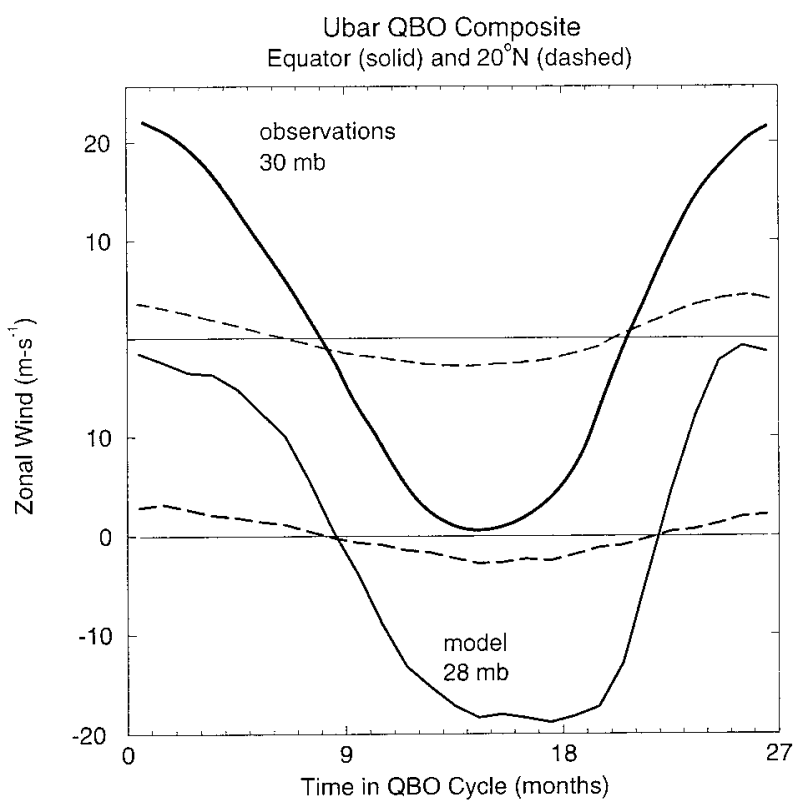

FIG. 7. Evolution of the zonal-mean wind through the composite QBO cycle. Results are presented for the equator (solid) and $20^{\circ} \mathrm{N}$ (dashed). The top panel is for observations at $30 \mathrm{mb}$ taken from Reed (1964). The bottom panel is for the present SKYHI model results at $28 \mathrm{mb}$.

$60^{\circ} \mathrm{C}$ colder than observed (near $1 \mathrm{mb}$ ). The $\mathrm{SH}$ winter circulation in the model also displays very little interannual variability.

\section{a. Extratropical zonal-mean fields}

Figure 9 shows the correlation coefficient between the seasonal zonal-mean $40-\mathrm{mb}$ equatorial zonal wind and the zonal-mean zonal wind at all heights and latitudes in the model. Results are presented for each of the four seasons December-February (DJF), MarchMay (MAM), June-August (JJA), and September-November (SON). Only contours for correlation coefficients with absolute value of at least 0.3 are plotted. For 45 independent samples the standard two-sided F-test gives a correlation of 0.288 as $95 \%$ significant. In each case the equatorial stratosphere displays regions of positive-negative-positive correlations stacked vertically, which simply reflect the presence of downward-propagating QBO wind reversals. Outside of the tropical stratosphere there are regions where the correlations are larger in magnitude than 0.3 , most notably in the $\mathrm{NH}$ polar region between $\sim 100 \mathrm{mb}$ and $0.1 \mathrm{mb}$ in DJF. The DJF correlation in Fig. 9a can be compared with Fig. 1 of Baldwin and Dunkerton (1991), which is based on geostrophic winds from $12 \mathrm{yr}$ of U.S. National Meteorological Center geopotential analyses. In these observations there is a region of positive correlation $>0.3$ at high latitudes, although it extends further equator- 

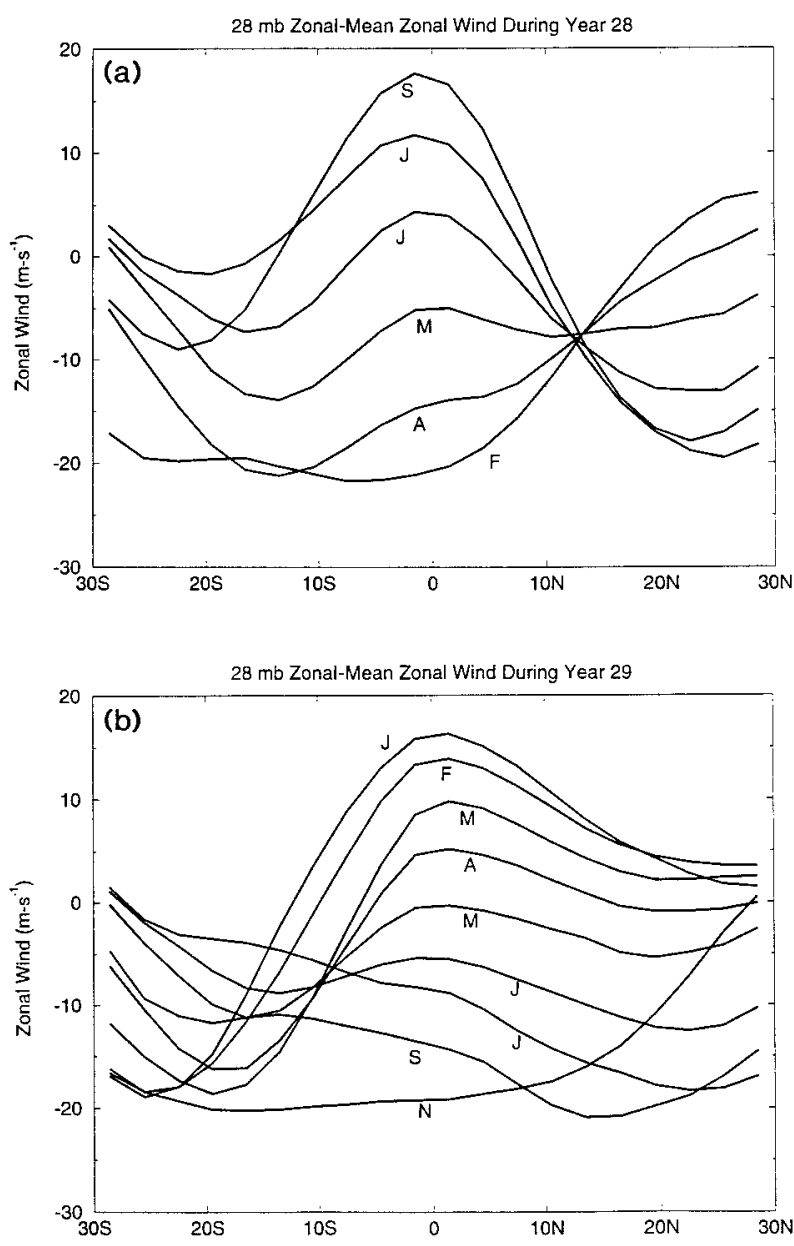

FIG. 8. The monthly averaged, zonal-mean zonal wind at $28 \mathrm{mb}$ for selected months during year 28 (a) and year 29 (b) of the SKYHI experiment.

ward (to about $45^{\circ} \mathrm{N}$ near $10 \mathrm{mb}$ ) than in the model result.

Figure 10 shows the DJF correlation of Fig. 9a computed using just the first $23 \mathrm{yr}$ (top) and then using only the last $22 \mathrm{yr}$ (bottom) of the $45-\mathrm{yr}$ record. The basic results for the Tropics and the high-latitude NH stratosphere are similar in the two periods, but there are certainly differences in the detailed patterns. This is most striking in the midlatitude mesosphere where the strong negative correlations seen in both hemispheres in the first $23 \mathrm{yr}$ are almost absent in the final $22 \mathrm{yr}$. The small region of positive correlation in the upper troposphere near $30^{\circ} \mathrm{S}$ in Fig. 9a is present in the first $23 \mathrm{yr}$ but not in the last 22 yr. This is an indication of how difficult it may be to determine stable statistics for the extratropical and tropospheric QBO effects based on the limited observational records that are currently available.

The east phase QBO versus west phase QBO differences can also be examined through compositing fields stratified by the sign of the tropical winds. When the time series of 45 consecutive DJF 40-mb equatorial zonal-mean zonal winds is examined, it turns out that there are 10 winters with $\bar{u}$ more than $11 \mathrm{~m} \mathrm{~s}^{-1}$ easterly than the 45-yr mean (which is $2.4 \mathrm{~m} \mathrm{~s}^{-1}$ easterly), and 10 winters with $\bar{u}$ more than $11 \mathrm{~m} \mathrm{~s}^{-1}$ westerly than the 45 -yr mean. Similarly there are 20 winters with $\bar{u}$ more than $3 \mathrm{~m} \mathrm{~s}^{-1}$ easterly than the $45-\mathrm{yr}$ mean, and 20 with $\bar{u}$ more than $3 \mathrm{~m} \mathrm{~s}^{-1}$ westerly than the $45-\mathrm{yr}$ mean. Figure 11 shows the east minus west phase zonal-mean wind composites based either on the 20 (top) or 40 (bottom) winters with most extreme $40-\mathrm{mb}$ equatorial winds. The reduction in the polar vortex strength by as much as $10 \mathrm{~m} \mathrm{~s}^{-1}$ in the east phase is quite apparent. It is interesting that in the extratropics the results in Figs. $11 \mathrm{a}$ and $11 \mathrm{~b}$ are quite similar, suggesting that extratropical QBO effects may not be much stronger when the equatorial $40-\mathrm{mb}$ wind is near an extreme than when the wind is just slightly easterly or westerly. This similarity between composites made using either the 20 most extreme DJF QBO phases or the 40 extreme DJF QBO phases is also seen in the analysis of the mean temperature and eddy fields discussed below.

Baldwin and Dunkerton (1991) and Dunkerton and Baldwin (1991) show east phase minus west phase zonal-mean zonal wind composites based on geostrophic winds from NMC observational analyses. Baldwin and Dunkerton (1991) present results from 12 winters of data (which extend up to $1 \mathrm{mb}$ ) and Dunkerton and Baldwin (1991) present results from 23 winters (extending only to $10 \mathrm{mb}$ ). The present model results (Fig. 11) are very similar to the 12-yr composite of Baldwin and Dunkerton (1991). The composite based on the longer series of Dunkerton and Baldwin (1991) shows stronger mean zonal wind contrasts between east and west QBO phases, with the maximum difference being $13 \mathrm{~m} \mathrm{~s}^{-1}$ at $10 \mathrm{mb}$ near $65^{\circ} \mathrm{N}$, compared with about 8 $\mathrm{m} \mathrm{s}^{-1}$ in Fig. 11.

Figure 12 shows the east phase minus west phase composited DJF zonal-mean temperature. This composite difference suggests that, on average, polar DJF temperatures between 100 and $10 \mathrm{mb}$ are $\sim 3^{\circ} \mathrm{C}$ warmer in the east QBO phase than in the west phase. At levels above $1 \mathrm{mb}$ the polar temperature signal is reversed in sign. This is consistent with the observed tendency for transient winter warmings of the polar stratosphere to be accompanied by cooling of the polar mesosphere (e.g., Andrews et al. 1987). Figure 13 shows the monthly mean North Pole temperature at the 28 -mb model level composited once again by equatorial 40-mb QBO phase. Results are presented for each individual month from October through April. The systematic effects of the tropical QBO on polar stratospheric temperature seem to be largely confined to the period December through March.

\section{b. Interannual variability}

The results presented in Figs. 9-13 leave little doubt that the model simulation in the high-latitude $\mathrm{NH}$ winter 

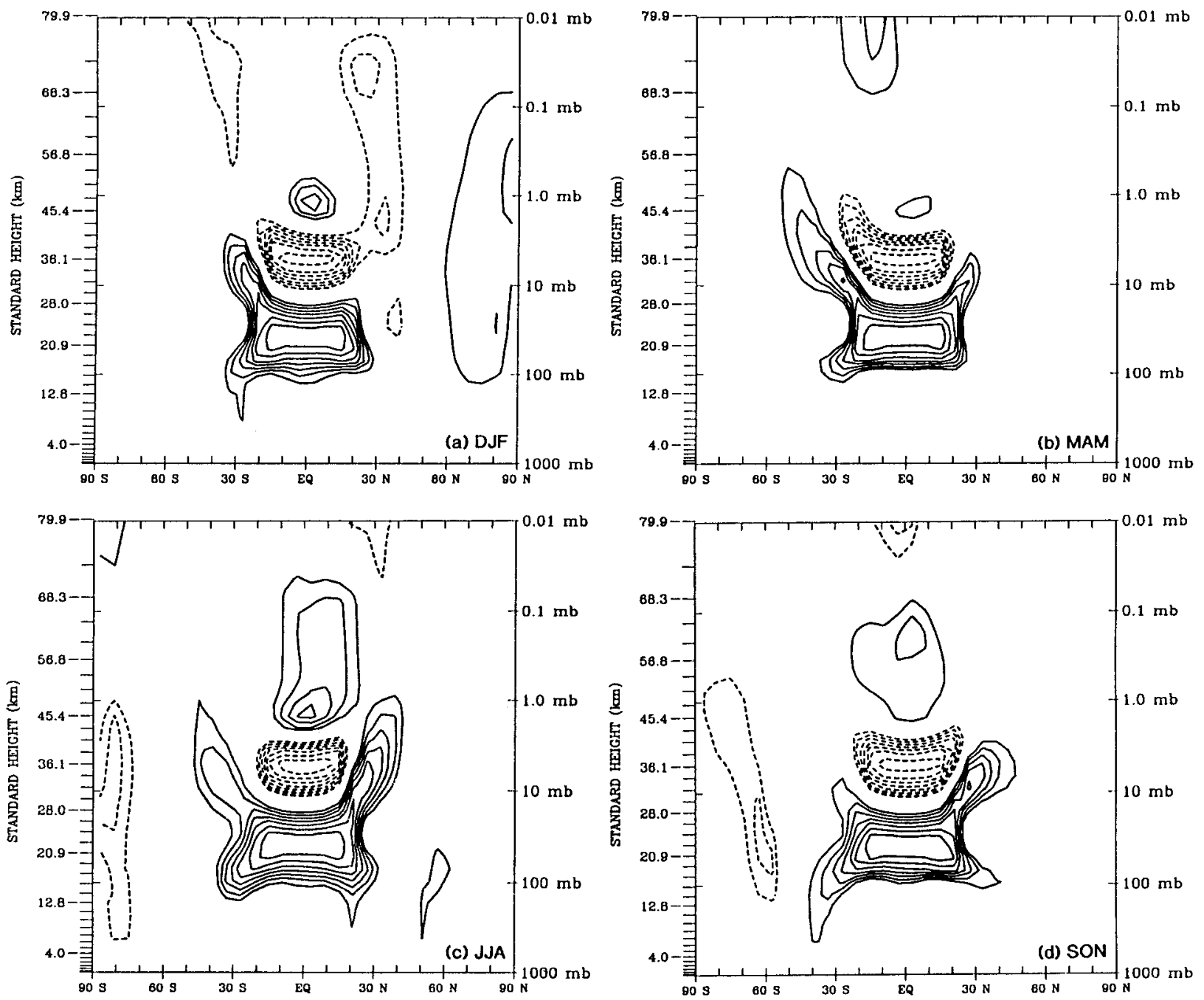

FIG. 9. Correlation coefficient for 45 -yr time series of seasonal-mean zonal-averaged wind with the equatorial wind at $40 \mathrm{mb}$. The contour interval is 0.1 , dashed contours denote negative values and only contours with absolute value greater than or equal to 0.3 are plotted. Results for (a) December-February, (b) March-May, (c) June-August, and (d) September-November.

stratosphere is significantly affected by the imposed tropical QBO. The question of how much the QBO contributes to the overall interannual variability of the extratropical stratosphere will now be addressed. Hamilton (1995a) compared the interannual variance of the monthly mean zonal-mean temperature in the 25 -yr control run of the SKYHI model with comparable observational results (up to $1 \mathrm{mb}$ ) based on NMC analyses (Randel 1992). The model results in the NH extratropics compared quite well with observations in January and February, but the control model significantly overestimated the interannual variability of the zonal-mean temperature in the high latitudes in December. The December standard deviation from the control run is reproduced here as Fig. 14a and the observational result of Randel as Fig. 14b. The overestimate of the temperature variability at high latitudes is apparent, particularly above $10 \mathrm{mb}$. It is unclear how typical this behavior may be for other middle atmosphere GCMs, but at least one model is known to have the opposite bias-that is, too little interannual variability in early winter (Manzini and Bengtsson 1996). Figure 14c shows the December standard deviation computed from the 45-yr QBO experiment. While the standard deviation is still much higher than observed, the QBO experiment produces somewhat less variability than in the control. The differences between the results in control and QBO experiments are much less in January or February (not shown). Figure 15 compares the standard deviation of DJF temperatures from the control run and the QBO experiment. Poleward of about $60^{\circ} \mathrm{N}$ the standard deviation of the DJF mean temperature in the upper stratosphere is reduced in the QBO experiment by $\sim 10 \%-$ $20 \%$. It seems that much of this reduction can be at- 

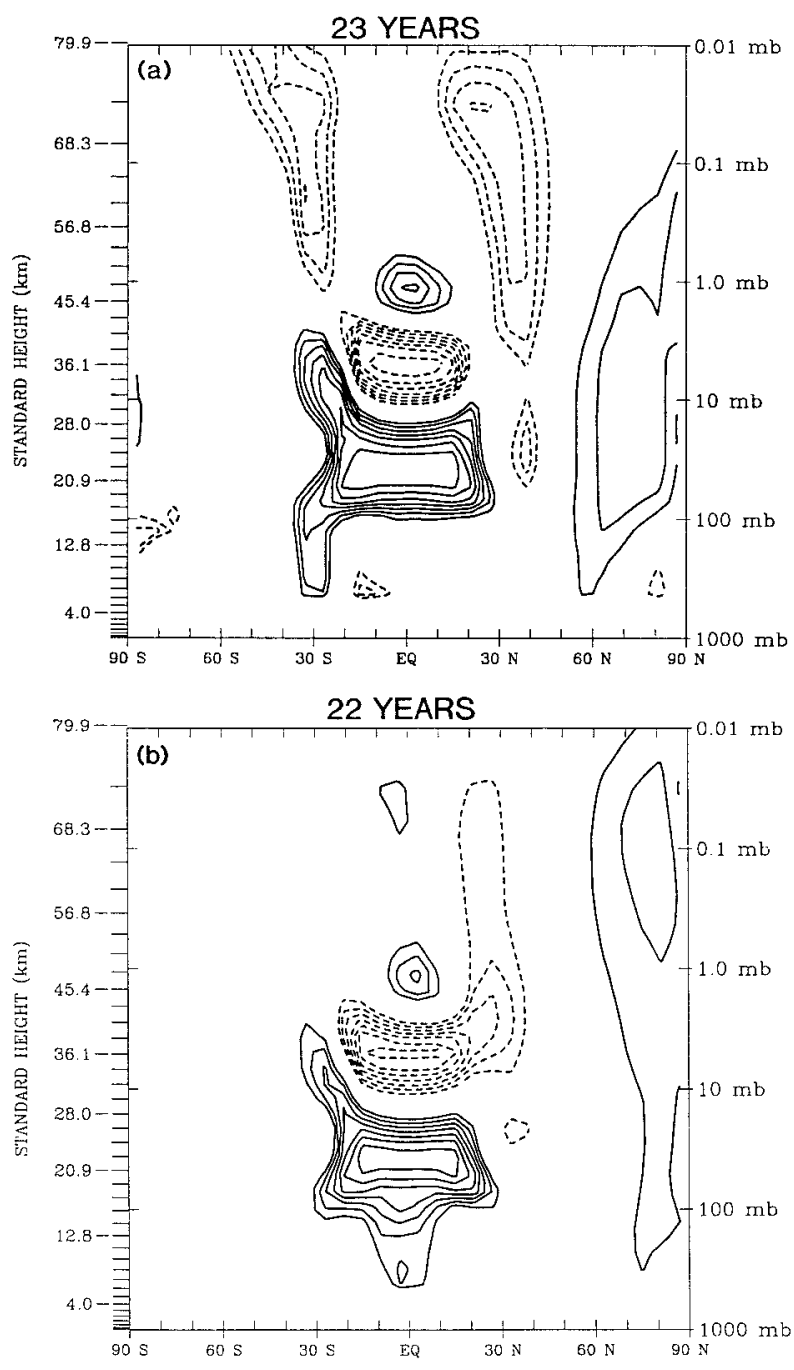

FIG. 10. As in Fig. 9a but using only the first $23 \mathrm{yr}$ of the time series (a), and the last $22 \mathrm{yr}(\mathrm{b})$.

tributed to a smaller variation among the winters with westerly winds at the equator. The standard deviation of the $1.08-\mathrm{mb}$ North Pole temperature in the 10 most easterly winters (judged by the $40-\mathrm{mb}$ equatorial wind) is $7.6^{\circ} \mathrm{C}$, but only $4.9^{\circ} \mathrm{C}$ for the temperature in the 10 most westerly winters.

While the total interannual variance of the winter extratropical circulation may actually be reduced by the inclusion of the QBO in the model, the tropical QBO can still act as a "pacemaker" for the extratropical variability. Figure 16 shows the time series of DJF North Pole temperatures plotted for $25 \mathrm{yr}$ of the control run and for the last $45 \mathrm{yr}$ of the QBO experiment. Inspection of this figure reveals a more pronounced biennial character to the polar temperature series with the imposed QBO, although the tendency for warm winters to be followed by cold winters (and vice versa) is far from perfect. In this respect the QBO experiment results ac-

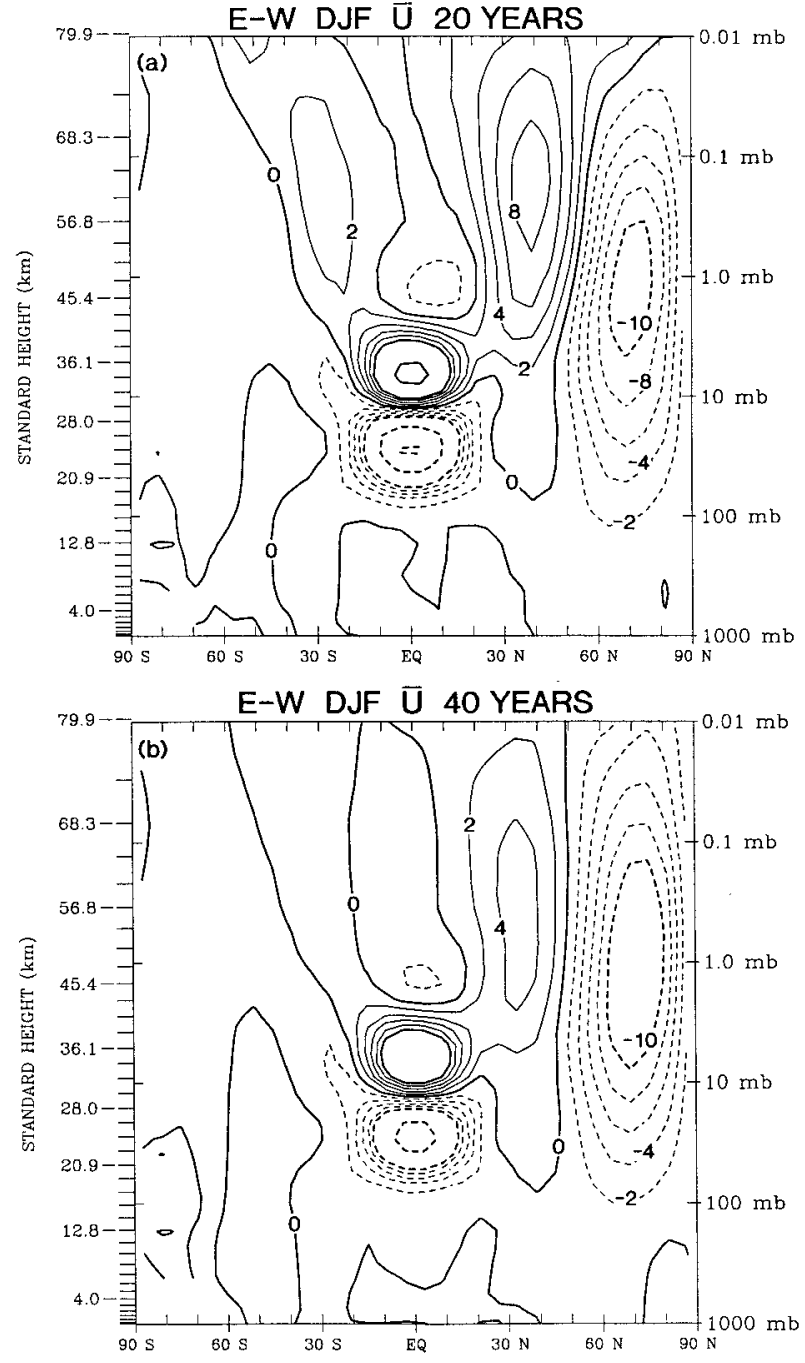

FIG. 11. East phase minus west phase composites of the DJF zonally averaged wind. Light contours at $\pm 2,4,6$, and $8 \mathrm{~m} \mathrm{~s}^{-1}$, heavy contours at $0, \pm 10,20, \ldots \mathrm{m} \mathrm{s}^{-1}$. Dashed contours denote negative values. (a) Result when the 10 extreme east phase and 10 extreme west phase winters are used. (b) Result when 20 east phase and 20 west phase winters are used.

tually look rather like comparable observational series (e.g., Fig. 1 of Dunkerton and Baldwin 1992). On the other hand, as noted earlier by Hamilton (1995a), the time series for the control model is apparently unrealistic in displaying sequences of as many as six anomalously cold or warm winters in a row.

\section{c. Sudden warmings}

The tendency for the observed NH winter-mean polar vortex to be weaker during the east $\mathrm{QBO}$ phase is associated, at least in part, with the more frequent occurrence of sudden warmings when there are mean easterlies in the tropical lower stratosphere (Labitzke 1982; 


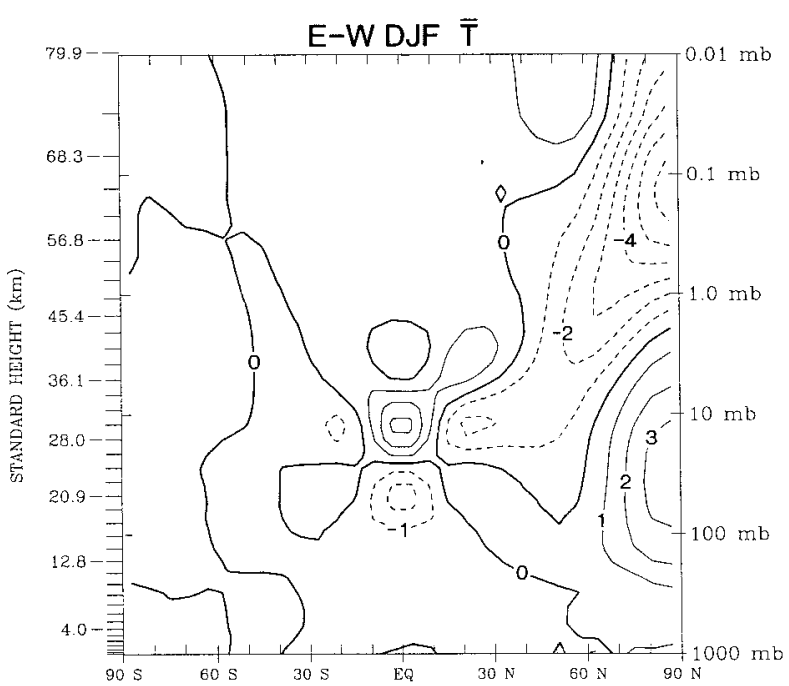

FIG. 12. East phase minus west phase composites of the DJF zonalmean temperature. The QBO phase is defined by the $40-\mathrm{mb}$ equatorial zonal wind and 20 extreme east phase and 20 extreme west phase winters are used. The contour interval is $1^{\circ} \mathrm{C}$ and dashed contours denote negative values.

Dunkerton et al. 1988). This same tendency is seen in the SKYHI model simulation with the imposed QBO. Figure 17 shows instantaneous profiles of the zonalmean equatorial wind in the model near the middle of each of $18 \mathrm{NH}$ strong sudden warming events. The 18 events selected had zonal-mean 9.2-mb temperature at $60^{\circ} \mathrm{N}$ colder than at the pole, and zonal-mean $9.2-\mathrm{mb}$ zonal winds easterly for at least two grid rows between $60^{\circ} \mathrm{N}$ and $80^{\circ} \mathrm{N}$. These conditions were met for at least 3 consecutive days in DJF. In addition, if two such warming periods occurred in the same winter, only the first warming date was used. The result of this was to select out the 18 events for which the equatorial winds are illustrated in Fig. 17. It can be seen that polar warm-

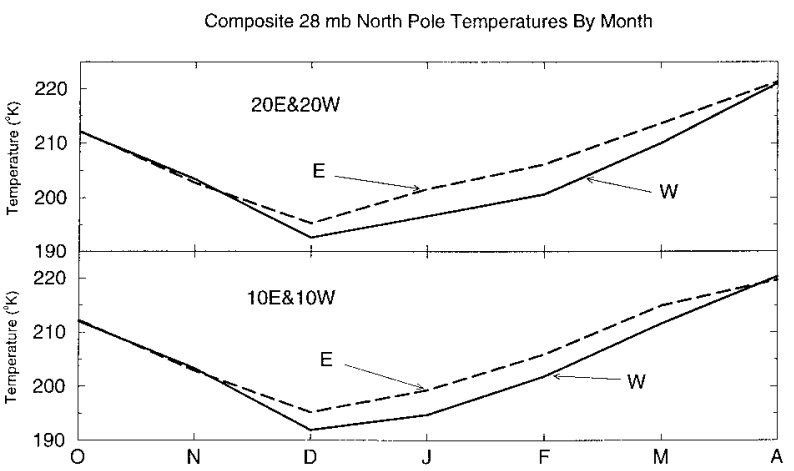

FIG. 13. The 28-mb North Pole temperature for each calendar month from October through April composited according to the phase of the 40-mb equatorial QBO in December-February. In the top panel the dashed (solid) curve is for the mean of $20 \mathrm{yr}$ of extreme east (west) phases. The curves in the bottom panel are for the mean of $10 \mathrm{yr}$ of extreme east (west) phases.
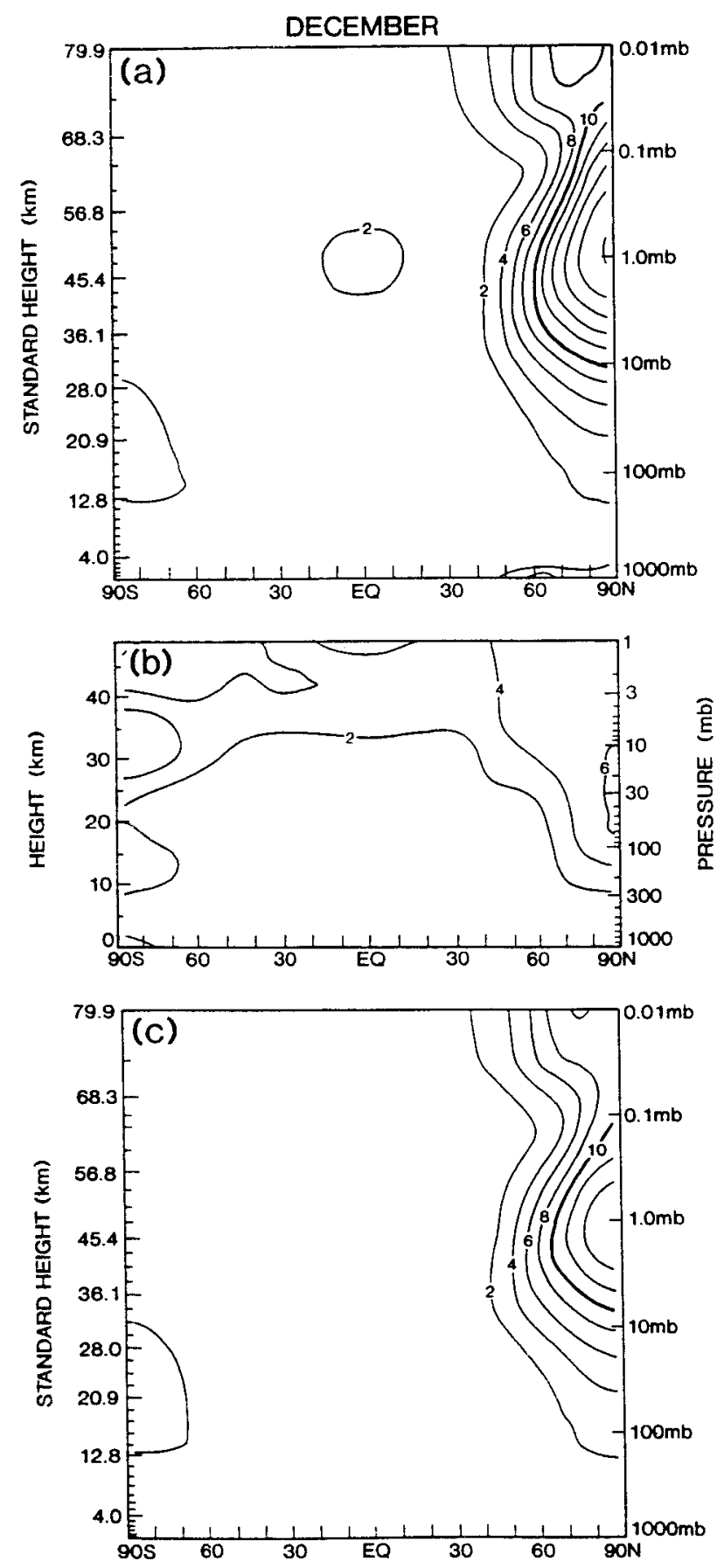

FIG. 14. The interannual standard deviation of zonal-mean December average temperatures: (a) from $25 \mathrm{yr}$ of the SKYHI control run, (b) from observations (Randel 1992), (c) from $45 \mathrm{yr}$ of the QBO experiment.

ings can occur with almost any tropical mean wind profile, but that the warmings are significantly more probable when the lower stratospheric equatorial wind is easterly. In fact, 13 (5) of the 18 warmings occurred during periods of equatorial easterly (westerly) winds 

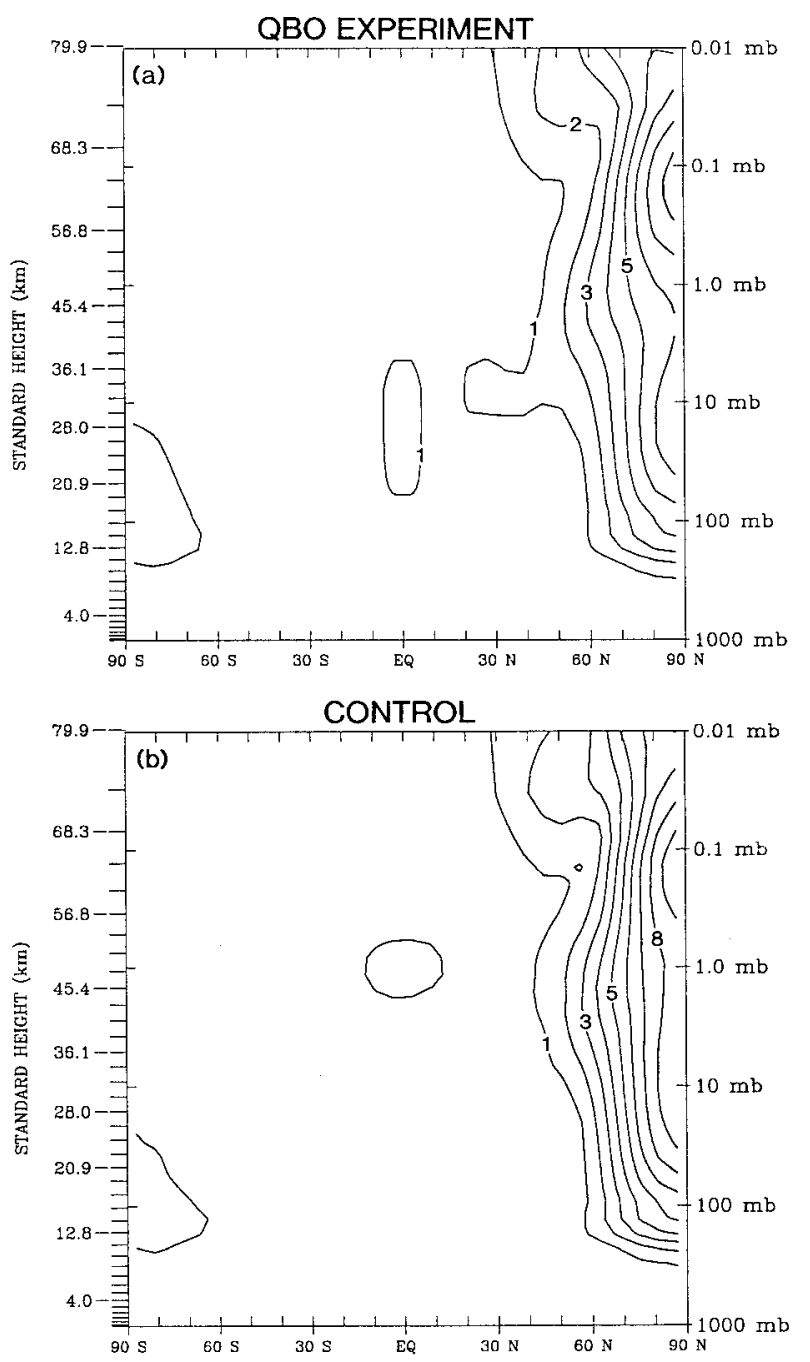

FIG. 15. (a) Standard deviation of the 45 -yr time series of DJF mean zonal-average temperature in the QBO experiment. Contour interval is $1^{\circ} \mathrm{C}$. (b) As in panel (a) but for $25 \mathrm{yr}$ of the control run of the model.

at $30 \mathrm{mb}$ (11 warmings when the easterlies exceeded $10 \mathrm{~m} \mathrm{~s}^{-1}$ ). The findings are similar if the winds at 40 $\mathrm{mb}$ or $50 \mathrm{mb}$ are used. This result seems quite consistent with the observations of Labitzke (1982) and Dunkerton et al. (1988) that major winter warmings are about twice as likely during the easterly QBO phase than during the westerly phase.

\section{d. QBO effects on extratropical stationary waves and wave driving of the mean flow}

As noted earlier, the observed changes in extratropical stratospheric mean flows are accompanied by changes in the quasi-stationary wave fields. Figure 18 shows east phase minus west phase composites of the DJF-mean geopotential at the 9-mb and 48-mb model levels (using 20 easterly and 20 westerly phase winters). The results

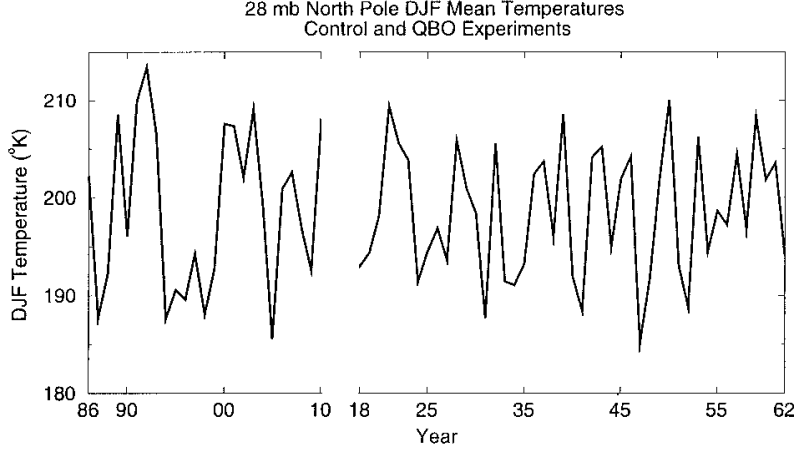

FIG. 16. Time series of the DJF 28-mb North Pole temperature for the control run (years marked 86-09) and for the QBO experiment (years marked 18-62).

can be compared to the same observational composites at $50 \mathrm{mb}$ and $10 \mathrm{mb}$ presented by Dunkerton and Baldwin (1991; their Figs. 3c and 3f) and based on 23 winters of NMC analyses. The zonal-mean component in both panels of Fig. 18 is consistent with the zonal wind composite shown in Fig. 11-that is, on average the polar low is less deep in the east QBO phase. At both levels the difference composite shows an elongation along an axis roughly $90^{\circ} \mathrm{E}-90^{\circ} \mathrm{W}$. A similar pattern (but shifted somewhat counterclockwise) is apparent in the observational composites of Dunkerton and Baldwin.

Figure 19 shows the east phase minus west phase composite of the DJF Eliassen-Palm (EP) flux divergence (expressed as a force per unit mass). There is a region of westward driving in this composite extending from at least $500 \mathrm{mb}$ up to $10 \mathrm{mb}$ at all extratropical $\mathrm{NH}$ latitudes. This is rather similar to the observed composite in Fig. 16a of Dunkerton and Baldwin (1991; note that their values are based on balanced winds used in the quasigeostrophic version of the EP flux). The fact that, on average, there is more westward forcing from eddies in the easterly QBO phase is consistent with the weaker polar vortex seen in this phase (Fig. 11). In the mean, the eddies in the model produce a westward forcing $\sim 1-2 \mathrm{~m} \mathrm{~s}^{-1}$ day $^{-1}$ in the extratropical NH lower stratosphere, and so the wave driving of the mean flow in this region is significantly augmented (reduced) in the easterly (westerly) QBO phase. In the NH upper troposphere there is also a significant augmentation of the westward forcing during the easterly QBO phase.

In the NH winter mesosphere the EP flux divergence (EPFD) in SKYHI is dominated by explicitly resolved vertically propagating gravity waves rather than largescale quasi-stationary eddies (e.g., Hamilton et al. 1995). The region of significant eastward forcing difference seen in the mesosphere poleward of $50^{\circ} \mathrm{N}$ in Fig. 19 can be attributed to the filtering effects of the mean flow in the polar vortex itself on the gravity waves (the upward propagation of eastward phase speed waves is diminished by a stronger vortex).

Figure 20 shows the DJF mean vertical EP flux com- 
ponent at $210 \mathrm{mb}$ (bottom) and $81 \mathrm{mb}$ (top), composited for the 10 most extreme east and west QBO phases. The vertical EP flux component is defined here as (Andrews et al. 1983):

$$
\mathrm{VEPF}=\cos \theta\left[\overline{u^{\prime} \omega^{\prime}}-\left(\frac{1}{a \cos \theta} \frac{\partial}{\partial \theta}(\bar{u} \cos \theta)-f\right) \frac{\left(\overline{v^{\prime} T^{\prime}}\right)}{\left(\kappa \frac{\bar{T}}{p}-\frac{\partial}{\partial p} \bar{T}\right)}\right]
$$

where $\theta$ is latitude, $\omega$ is vertical velocity (pressure coordinates), $f$ is the Coriolis parameter, $v$ is the south wind component, $T$ is temperature, $\kappa$ is ratio of gas constant to specific heat, and $p$ is pressure. Over the $20^{\circ}-70^{\circ} \mathrm{N}$ latitude range shown, the upward EP flux is larger on average in the east phase than the west phase. The figure also shows a rough measure of the sampling error in the determination of the 10 -yr mean, $\pm 2 \sigma /(n$ $-1)^{1 / 2}$ - that is, \pm 2 times the standard deviation of the 10 individual DJF means divided by $(n-1)^{1 / 2}$, where $n=10$. By this simple measure, the differences between the east phase and west phase composite VEPF appear to be significant at least over some latitude ranges. This is consistent with the notion that the EP flux is more focused into the polar stratosphere during the tropical easterly QBO phase. It is interesting that this effect extends right into the upper troposphere. The sign of the difference between the east and west phase composite VEPF in the extratropical NH reverses at and above $\sim 2 \mathrm{mb}$. This presumably reflects the filtering of the gravity wave field by the mean flow, and is consistent with the mesospheric results for the EPFD shown in Fig. 19.

\section{e. Summary}

In summary, the $\mathrm{NH}$ winter extratropical circulation in the model responds significantly to the imposed tropical QBO. The strongest indications of extratropical response seem to occur when data are stratified using the phase of the equatorial QBO at model levels near 40 $\mathrm{mb}$. In general the polar regions are warmer, the vortex is weaker, and midwinter sudden warmings are more frequent in the easterly QBO phase. These effects are in at least qualitative agreement with observed results. The detailed comparison of the model results with observations is complicated by the limited length of available observational records. Indeed, it was shown that the present model results for extratropical QBO effects are somewhat different in the first and second half of the $45 \mathrm{yr}$ of integration analyzed.

\section{Tropospheric effects}

As noted in the introduction, some researchers have claimed to find evidence for tropospheric influence of the stratospheric QBO. Such effects could arise through several possible mechanisms. The changes induced in the mean flow in the polar stratosphere could also affect the troposphere through downward reflection of the component of planetary wave activity that is directed upward into the polar region. The fact that the VEPF even at $210 \mathrm{mb}$ is affected by the tropical QBO phase (Fig. 20) may be suggestive in this regard. However, just the redistribution of the EPFD in the stratosphere in response to the QBO could affect the zonal-mean state of the extratropical troposphere through the induced meridional circulation (e.g., Haynes et al. 1991). In the Tropics there has been speculation that the wind shears and temperature changes associated with the QBO near the tropopause could influence deep convection, thus producing a link between the phase of the stratospheric QBO and tropical rainfall (Mukherjee et al. 1985; Gray et al. 1992a), or even hurricane frequency (Gray 1984; Gray et al. 1992b).

The results shown earlier (Figs. 9, 10, and 11) suggest that the QBO influence on the NH winter polar vortex extends down to tropospheric levels. Figure 21 shows the DJF zonal-mean 210-mb zonal wind composited by QBO phase and presented in a format similar to that of Fig. 20. The east phase-west phase differences are well under $1 \mathrm{~m} \mathrm{~s}^{-1}$ everywhere except near $50^{\circ}-70^{\circ} \mathrm{N}$. Poleward of about $50^{\circ} \mathrm{N}$ the westerly vortex is stronger on average by as much as $1 \mathrm{~m} \mathrm{~s}^{-1}$ in the westerly QBO phase, and the difference between easterly and westerly phases is near the $\pm 2 \sigma /(n-1)^{1 / 2}$ threshold. This result is consistent with the observations of Dunkerton and Baldwin (1991). It is seen also in the model results at midtroposphere (say in a 500-mb composite). This effect seems strong enough that it could possibly contribute slightly, but usefully, to the skill of seasonal weather forecasts.

Attempts to find any association between the tropical rainfall and the phase of the $\mathrm{QBO}$ in the simulation have been quite unsuccessful. The zonal-mean precipitation (not shown) was almost identical in the east and west phase composites, despite the fact that the difference of the zonal wind composites based on the 40-mb extremes (Fig. 11) does show considerable shear in the lower stratosphere. Figure 22 shows the east and west phase composited DJF precipitation averaged over the six grid 


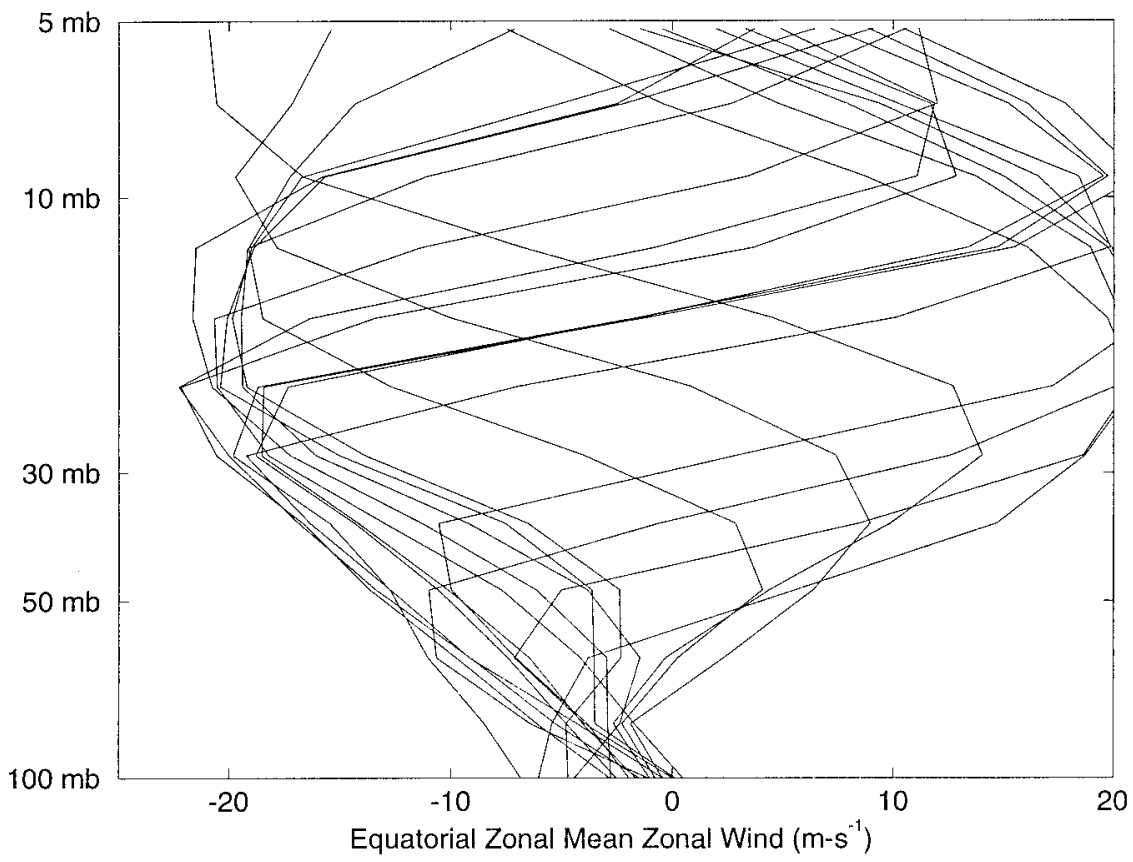

FIG. 17. Instantaneous profiles of the equatorial zonal-mean zonal wind near the middle of each of 18 strong sudden warmings in the SKYHI QBO experiment.

rows nearest the equator, and presented in a format similar to that shown for the zonal-mean composites in Figs. 20 and 21. The difference between the east phase and west phase composite rainfall seems to be quite small almost everywhere, although at some longitudes it does approach or exceed the $\pm 2 \sigma /(n-1)^{1 / 2}$ limits. There seems to be no coherent geographical pattern to the differences - a point that is emphasized when maps of east phase minus west phase rainfall differences (not shown) are examined.

The GCM experiment described here should provide a clean test of the possibility of stratospheric QBO influence on the troposphere. Unlike analysis of real world data, the present model has a tropical stratospheric QBO that is not significantly influenced by tropospheric variability. In addition, other sources of tropospheric interannual variability (notably those associated with SST changes) are suppressed in the model, thus allowing any QBO effects to stand out more clearly. The lack of significant tropical tropospheric effects in this model experiment would argue against proposals to use the stratospheric QBO in seasonal weather predictions for the Tropics (e.g., Gray 1984). Of course, the model does have significantly simplified parameterized processes (notably moist convection) that make impossible any firm conclusion in this regard.

\section{Tropical stratospheric momentum budget}

As noted earlier, the control model does not generate anything approaching a realistic QBO in the tropical stratosphere. This problem is shared by other GCMs.
The QBO in the real atmosphere is thought to be driven largely by the EPFD associated with vertically propagating waves (e.g., Lindzen and Holton 1968; Holton and Lindzen 1972), although the exact contribution of waves of various scales and frequencies has not been determined from observations. Possible explanations for the lack of a QBO in GCM simulations include a meanflow wave driving that is unrealistically weak, an unrealistically strong subgrid-scale dissipation, or possibly problems with the numerical representation of the wave-mean flow interaction with relatively crude vertical resolution. One motivation for the present experiment was to see how the waves (and other aspects of the model dynamics) in the tropical region itself respond to the presence of a realistic mean wind profile at low latitudes. This section reports on an analysis of the zonal-mean zonal momentum budget of the near-equatorial stratosphere in the QBO experiment.

The important aspects of the zonally averaged dynamics can be reconstructed from the zonal-mean quantities archived once per day throughout the integration. This allowed the data to be composited over a 27-month cycle. The full 45-yr record was divided into twenty 27-month segments and an average over the 20 cycles was then performed. Figure 23 shows the monthly mean zonal-mean zonal wind plotted at 2-month intervals through the composited QBO cycle. Also shown is the monthly mean acceleration of the zonal wind. The values displayed are averages over the four model grid rows closest to the equator $\left(4.5^{\circ} \mathrm{S}-4.5^{\circ} \mathrm{N}\right)$. In month 1 of the composite the easterly phase is well developed at and above $10 \mathrm{mb}$. Over the next year the easterly maximum 

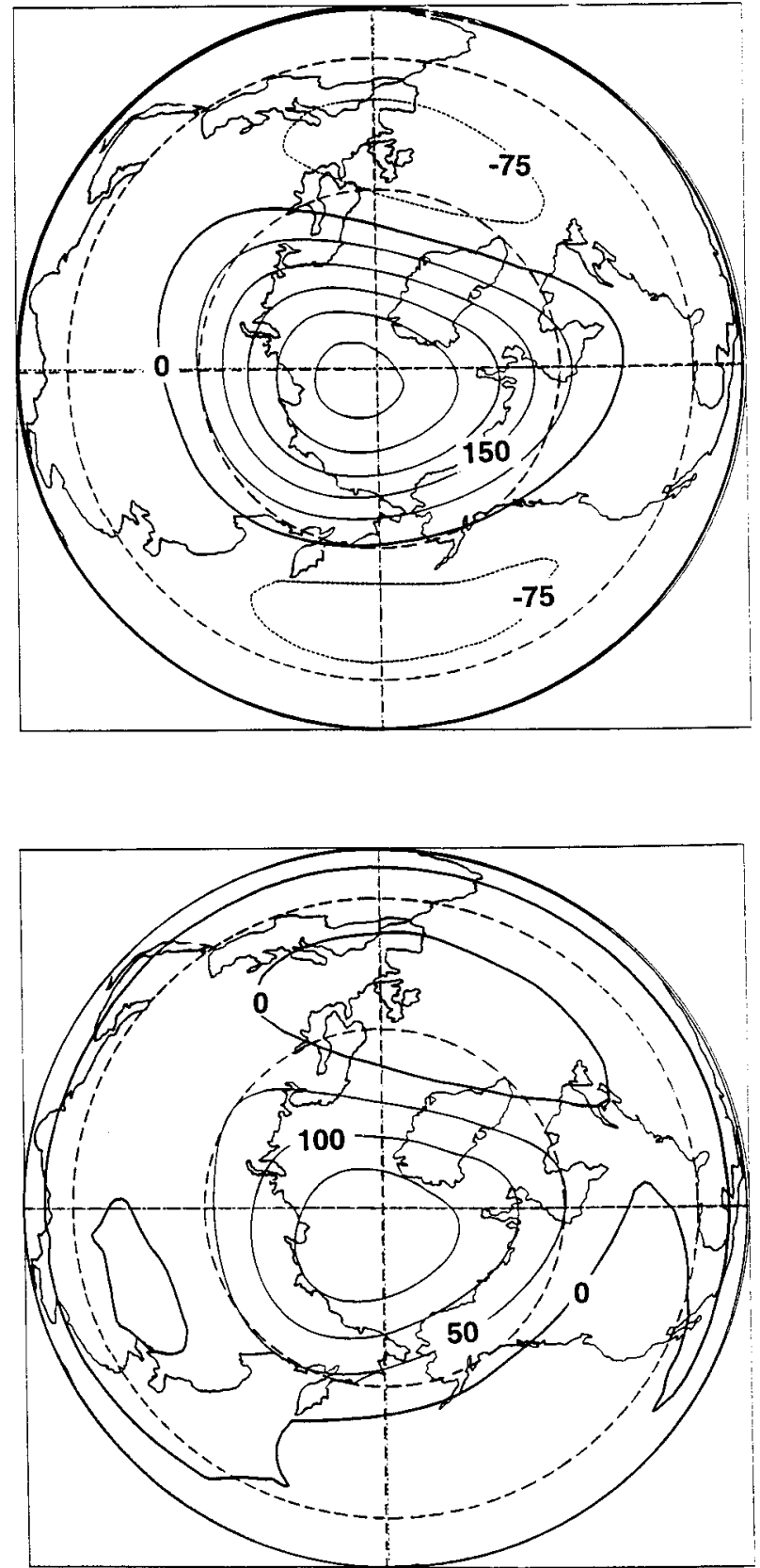

FIG. 18. East phase minus west phase composites of the DJF geopotential height at (top) $9 \mathrm{mb}$ and (bottom) $48 \mathrm{mb}$. The QBO phase is defined by the $40-\mathrm{mb}$ equatorial zonal wind and 20 extreme east phase and 20 extreme west phase winters are used. The contour interval is $75 \mathrm{~m}$ in the top panel and $50 \mathrm{~m}$ in the bottom panel. Dashed contours denote regions where the heights are lower in the easterly phase.

descends while a westerly phase develops at higher levels. During the last year of the composite the westerly maximum descends and the easterly phase develops higher up.

Figure 24 shows the total EPFD and the EPFD calculated considering only the vertical component of the

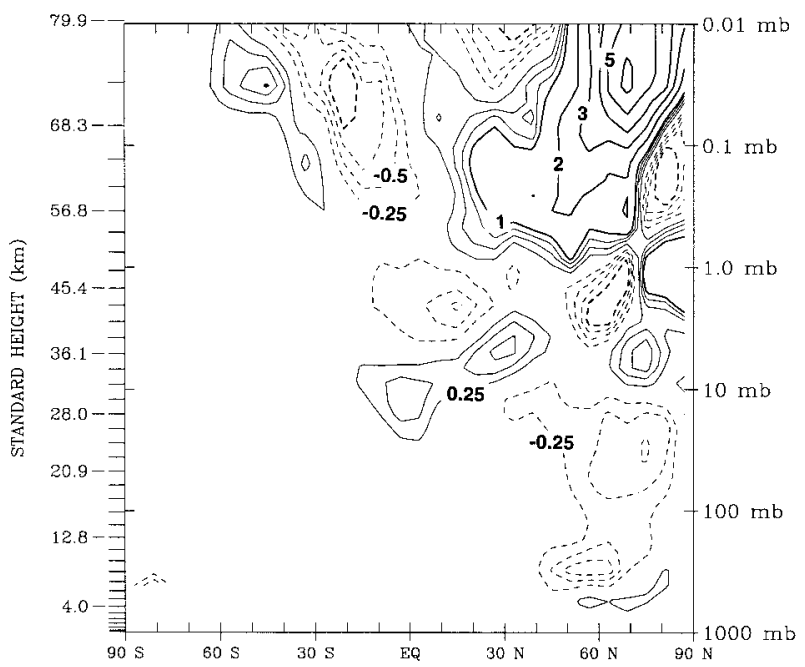

FIG. 19. East phase minus west phase composite of the DJF Eliassen-Palm flux divergence. Light contours are at $\pm 0.25,0.5$, and 0.75 $\mathrm{m} \mathrm{s}^{-1}$ day $^{-1}$, and heavy contours are at $\pm 1,2,3, \ldots \mathrm{m} \mathrm{s}^{-1}$ day $^{-1}$. No zero contour is plotted and (solid) dashed contours denote regions of enhanced eastward (westward) mean flow driving during the east QBO phase.

EP flux (VEPFD) for four months through the composite QBO cycle. These terms are compared to the actual zonal-mean zonal acceleration. The striking aspect of panels $\mathrm{a}, \mathrm{b}$, and c (corresponding to months 11,15 , and 23 of the composite) is that in the region of strongest acceleration the EPFD is actually larger than the realized acceleration. This seems to be true for the westerly acceleration phase, and for the easterly acceleration phase at high altitudes (say above $20 \mathrm{mb}$ ). The result for month

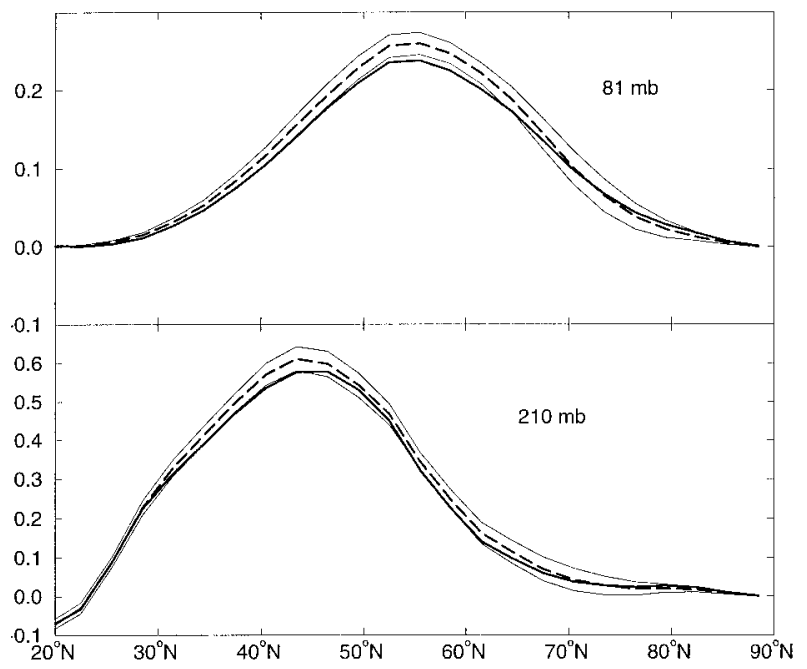

FIG. 20. DJF mean vertical EP flux averaged for the $10 \mathrm{yr}$ of extreme west QBO phase (heavy solid) and for the $10 \mathrm{yr}$ of extreme east QBO phase (heavy dashed). The light solid lines are plotted at $\pm D$ of the east phase mean, where $D$ is two-thirds of the standard deviation of the 10 individual values. Results for (top) $81 \mathrm{mb}$ and (bottom) $210 \mathrm{mb}$. (values in $\mathrm{Pa} \mathrm{m} \mathrm{s}{ }^{-2}$ ). 


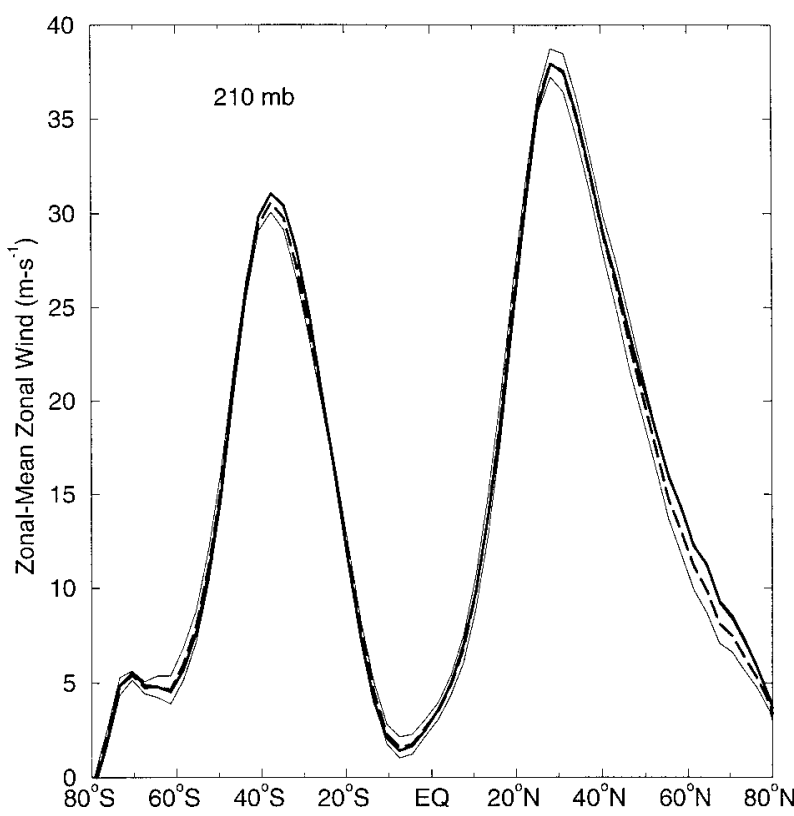

FIG. 21. DJF zonal-mean zonal winds at $210 \mathrm{mb}$ averaged for the $10 \mathrm{yr}$ of extreme west QBO phase (heavy solid) and for the $10 \mathrm{yr}$ of extreme east QBO phase (heavy dashed). The light solid lines are plotted at $\pm D$ of the east phase mean, where $D$ is two-thirds of the standard deviation of the 10 individual values.
3 in panel $24 \mathrm{~d}$ is much different, however. Here, the peak easterly accelerations below $10 \mathrm{mb}$ are not matched by the EPFD, and the VEPFD actually opposes the realized acceleration.

Figure 24 seems to indicate that the model has enough vertically propagating wave activity to account for much of the required QBO accelerations. The VEPFD term changes strongly through the QBO cycle in a manner consistent with that expected from simple models of wave-mean flow interaction (e.g., Lindzen 1971, 1972; Holton and Lindzen 1972). For example, in month 11 of the composite there is a strongly developed easterly phase below about $10 \mathrm{mb}$ and an incipient westerly phase above. The presence of the easterlies in the lower stratosphere should allow the efficient propagation of eastward waves through this layer and the generation of an eastward VEPFD at higher levels. This is actually seen in the results in Fig. 24a.

Figure 25 shows the zonal momentum budget contributions from the subgrid-scale horizontal diffusion of momentum and from the advection and Coriolis torque associated with the residual meridional circulation. Again these terms are compared with the realized zonalmean accelerations. The horizontal diffusion makes only a very minor contribution, but the advection from the residual circulation is quite large, and is usually opposed to the realized accelerations, particularly in the 50-5$\mathrm{mb}$ region. This can be understood as a consequence of the large-scale upwelling expected in the lower stratosphere. The forces causing the descent of the QBO shear zones have to overcome this upward advection. Diabatic

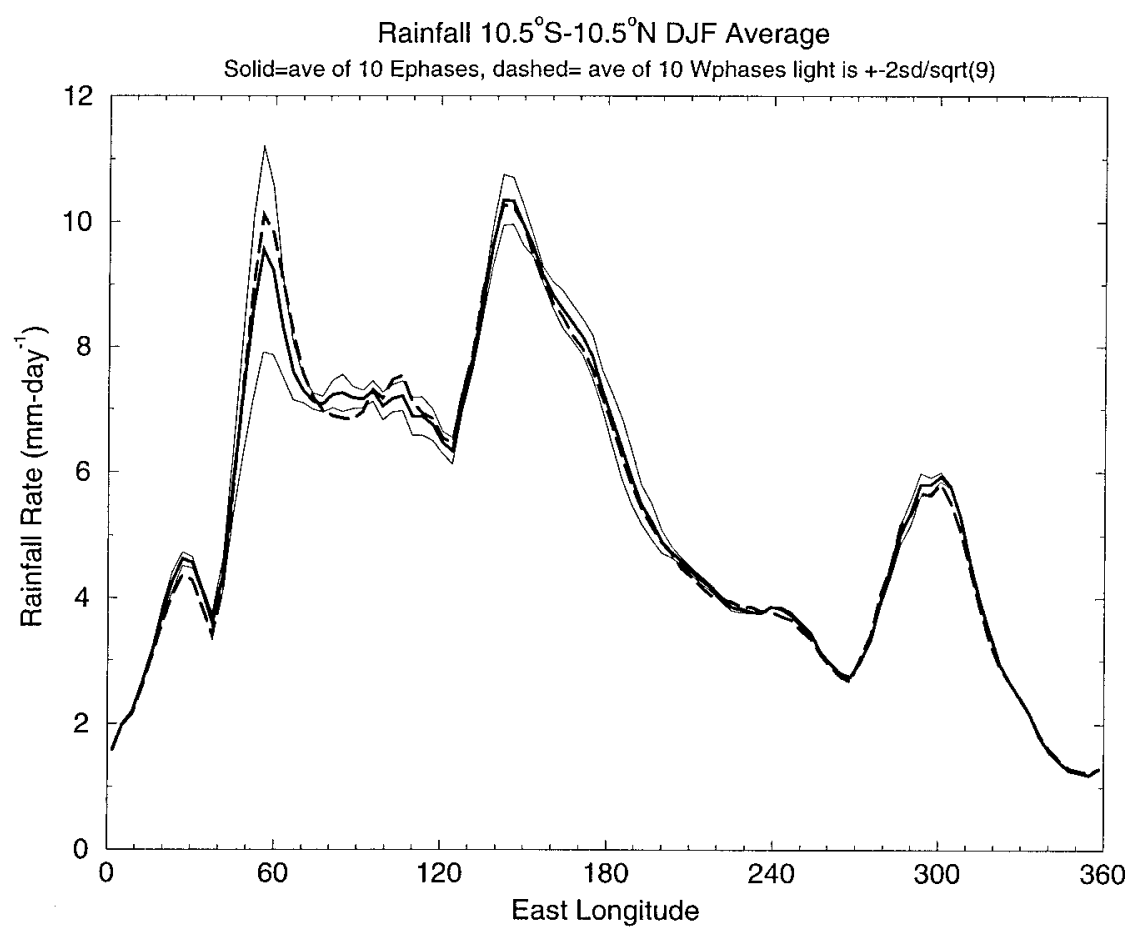

FIG. 22. As in Fig. 20 but for the DJF-mean rainfall averaged between $10.5^{\circ} \mathrm{S}$ and $10.5^{\circ} \mathrm{N}$. 

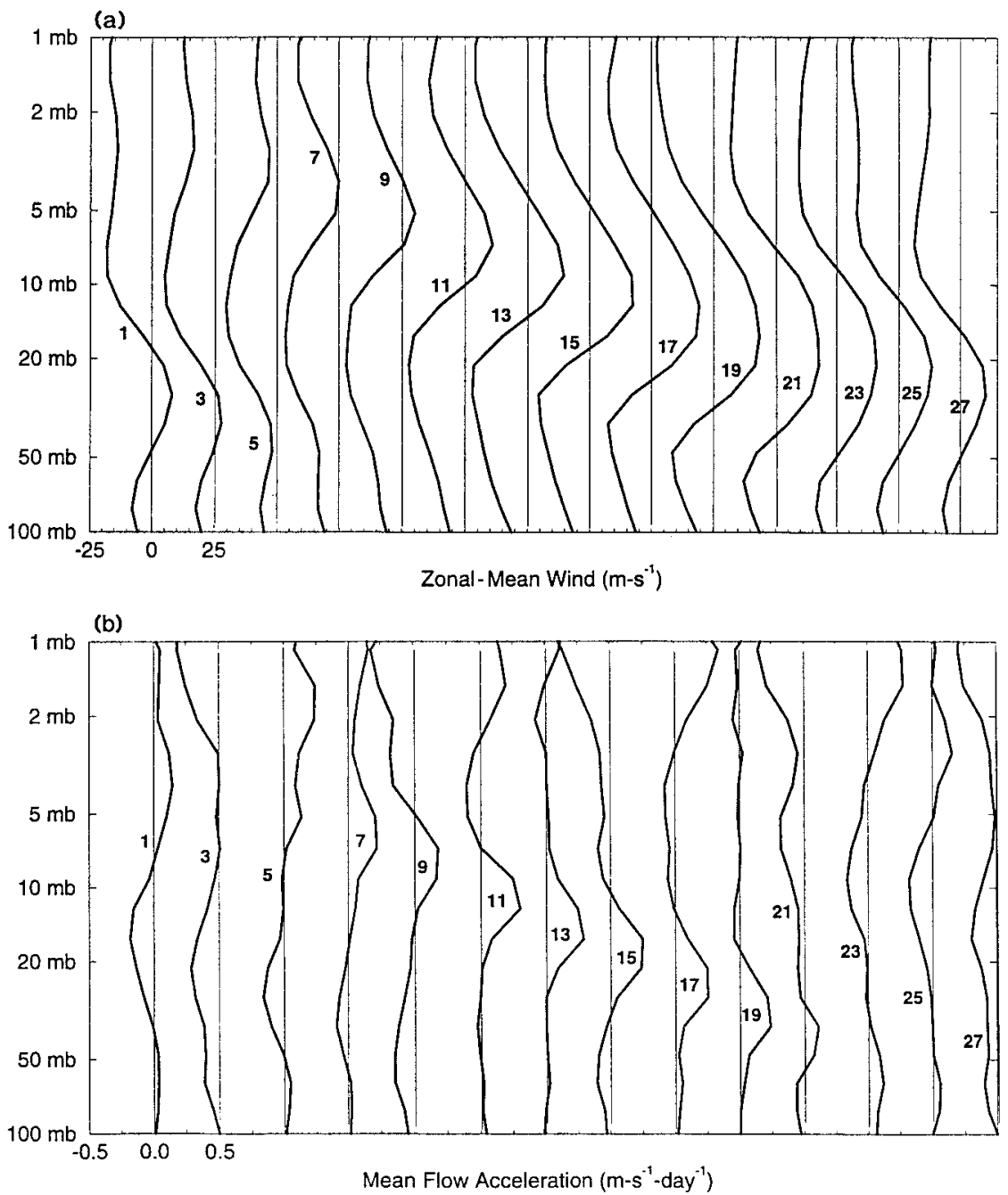

FIG. 23. (a) The zonal-mean zonal wind averaged over $4.5^{\circ} \mathrm{S}-4.5^{\circ} \mathrm{N}$ for individual months in the 27-month QBO composite. Successive curves are plotted for months 1, 3, 5, 7, ., 27. (b) As in panel (a) but for the rate of change of the zonal-mean zonal wind.

heating rates for the model near the equator between 50 and $5 \mathrm{mb}$ vary between $\sim 0.2$ and $0.5^{\circ} \mathrm{C}$ day $^{-1}$, corresponding to upward velocities of $\sim 600-1500$ meters per month [these are at least roughly consistent with various observational estimates of the equatorial upwelling in the real stratosphere (e.g., Holton et al. 1995)]. These values are comparable to the descent rates of the QBO shear zones, and so it is not surprising that the advection by the residual circulation provides a significant opposition to the realized accelerations. Above $5 \mathrm{mb}$ the cross-equatorial component of the residual circulation starts to become a significant factor, and the negative correlation between accelerations and the residual advection term is not so evident.

Figure 26 shows the zonal momentum budget contributions from the vertical subgrid-scale momentum diffusion and the imposed forcing, once more compared with the realized accelerations. The vertical diffusion term tends to be fairly small, except in the regions of strong westerly shear, for example, between $5 \mathrm{mb}$ and $20 \mathrm{mb}$ in month 11 (Fig. 26a) and between $10 \mathrm{mb}$ and $30 \mathrm{mb}$ in month 15 (Fig. 26b). At these times the diffusion acts strongly to smooth out the curvature in the mean wind profile, resulting in the oscillatory contribution to the momentum forcing seen in Figs. 26a,b. This effect augments the westerly acceleration at the bottom of the westerly shear region and reduces it above, thus contributing to the downward propagation of the westerlies.

The contrast between the behavior for the vertical diffusion term in the easterly and westerly shear layers is a consequence of the stronger peak westerly shears noted earlier. The subgrid vertical mixing in the model is treated as a second-order diffusion with coefficient 

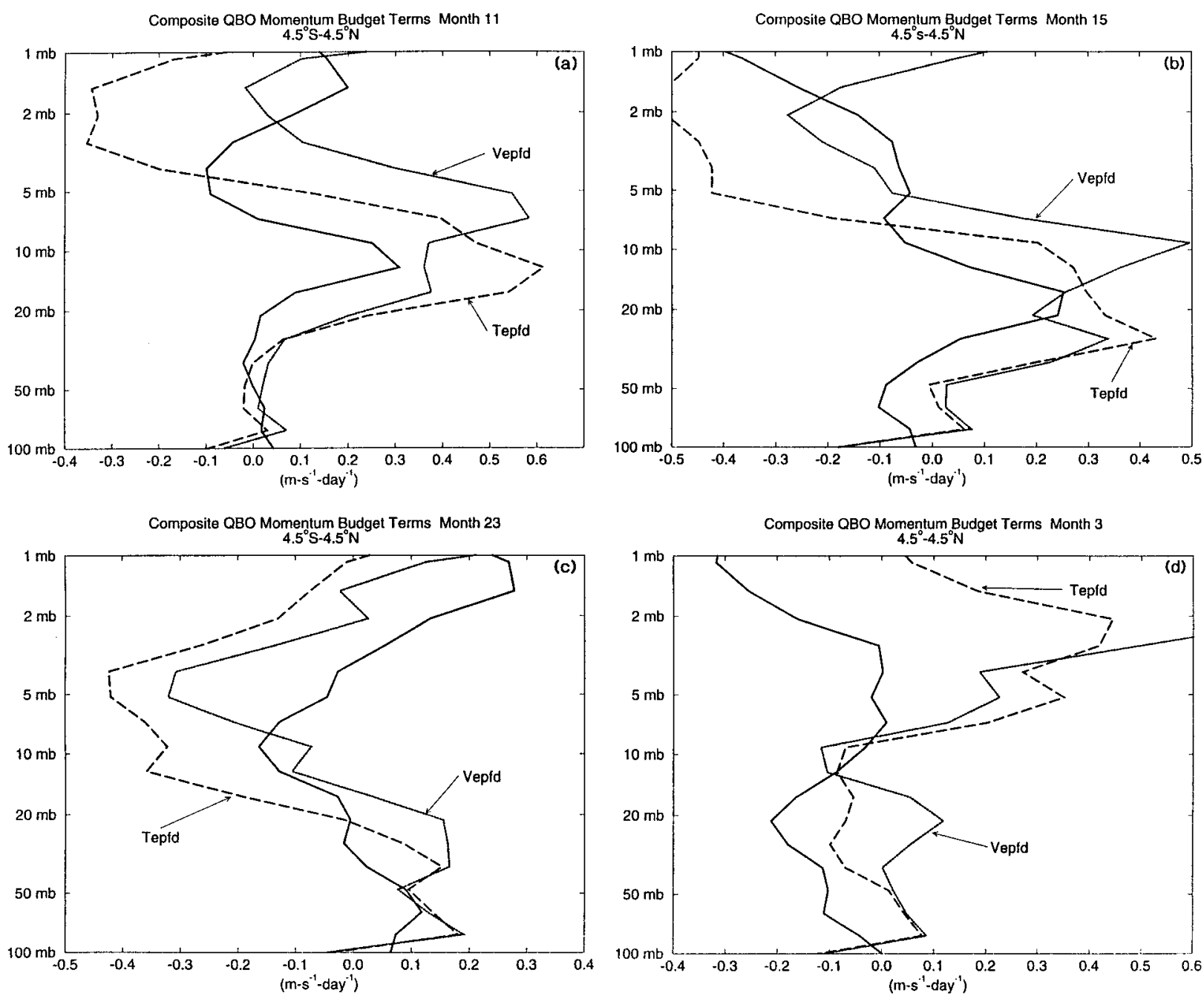

FIG. 24. Components of the zonal-mean zonal momentum budget averaged over $4.5^{\circ} \mathrm{S}-4.5^{\circ} \mathrm{N}$ for individual months in the $27-\mathrm{month}$ QBO composite. In each panel the solid curve shows the rate of change of the zonal wind, the long dashed curve is the total Eliassen-Palm flux divergence (EPFD) and the dotted curve is the EPFD computed just from the vertical EP flux. Results for (a) month 11, (b) month 15, (c) month 23 , and (d) month 3 . Note the horizontal axis is slightly different for each plot.

strongly dependent on the resolved Richardson number, $\mathrm{Ri}$ (Levy et al. 1982). The expression for the diffusivity coefficient is

$$
\begin{array}{ll}
K_{v}=K_{m} & \text { if } \mathrm{Ri}>A / 4 \\
K_{v}=K_{m}+l^{2} S(1-4 \mathrm{Ri} / A)^{0.5} & \text { if } 0<\mathrm{Ri}<A / 4 \\
K_{v}=K_{m}+l^{2} S & \text { if } \mathrm{Ri}<0,
\end{array}
$$

where $K_{m}$ is the molecular viscosity (negligible in the height range of present interest), $l$ is the mixing length (taken to be a constant $30 \mathrm{~m}$ ), and $\mathcal{S}$ is the magnitude of the vertical shear. The factor $A$ is designed to account for the subgrid-scale inhomogeneity in the vertical shear and is taken to be

$$
A=1+0.1(10 \Delta z)^{1.5}
$$

where $\Delta z$ is the spacing between adjacent layers in $\mathrm{km}$. This factor approaches unity when the level spacing becomes small, and allows instability at larger resolved $\mathrm{Ri}$ when the level spacing is large. For grid-spacing of $2 \mathrm{~km}$ the mixing will turn on when the resolved Ri drops below 2.5. In the present $\mathrm{QBO}$ experiment, the strongest westerly mean flow shears can be $\sim 0.01 \mathrm{~s}^{-1}$, which results in a Ri computed from the zonal mean fields $\sim 2$. Thus the mixing is likely turned on almost all the time in these very strong shear layers. The peak easterly shears are considerably $(\sim 50 \%)$ weaker and so the mixing is more sporadic. This formulation of the vertical diffusion was devised by Levy et al. (1982) in order to take into account the presence of continuous spectrum of waves in the vertical, and it is still an open question as to whether it is appropriate in the presence of very strong mean flow shears.

The imposed forcing term plotted in Fig. 26 is somewhat complicated in its evolution through the QBO cy- 

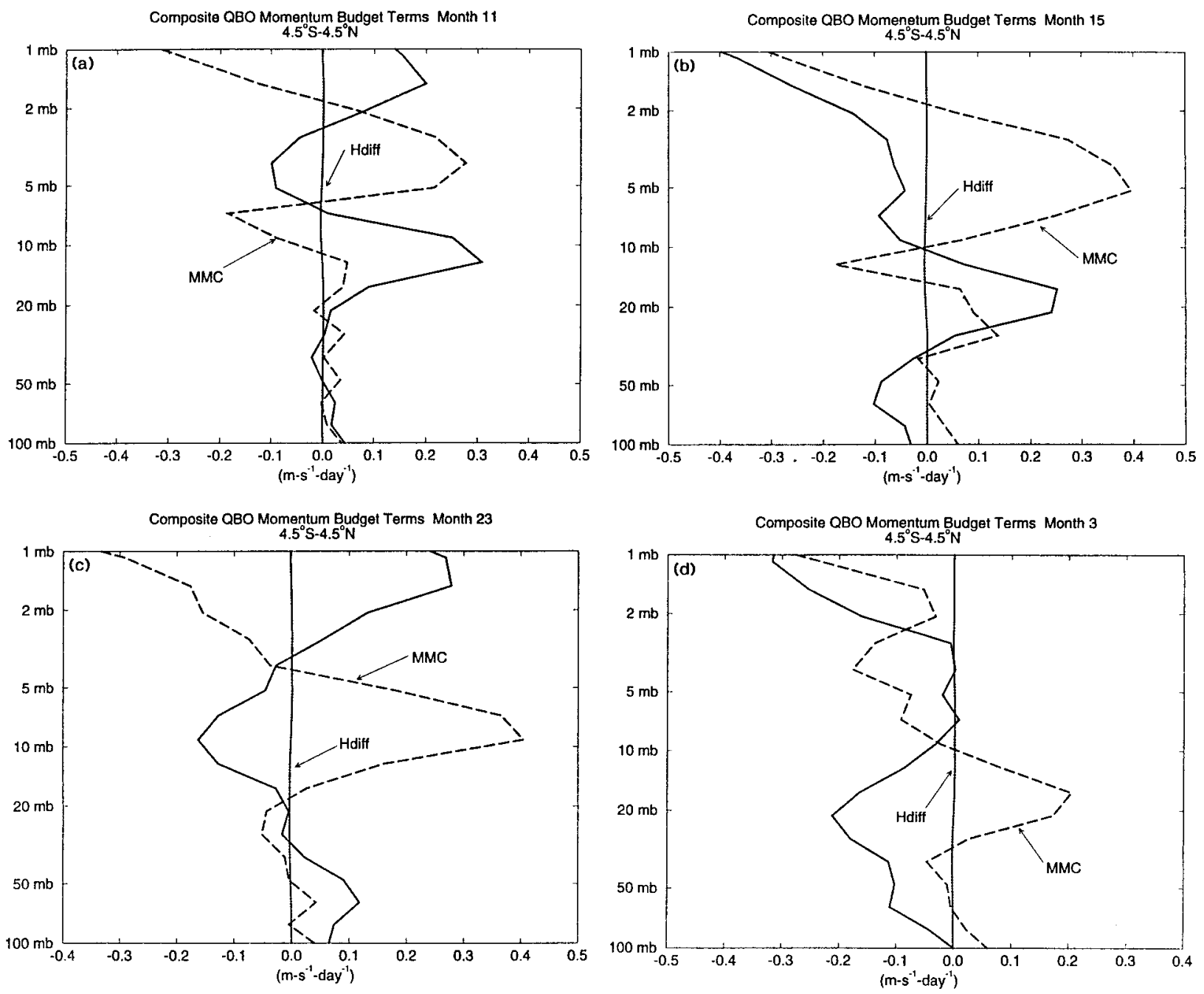

FIG. 25. As in Fig. 24 but showing some different components of the momentum budget. As in Fig. 24 the solid curve shows the rate of change of the zonal wind. The long-dashed curve shows the contribution from the residual meridional circulation and the dotted curve shows the contribution from the horizontal subgrid-scale diffusion of momentum.

cle. In the vicinity of the peak westerly shears the forcing largely cancels out the vertical diffusion term. In the peak easterly accelerations in months 23 and 3 (Figs. $26 \mathrm{c}, \mathrm{d})$ the imposed forcing is in the same sense as, and somewhat stronger, than the realized accelerations. In these cases the extra forcing apparently is necessary to supplement the resolved wave driving to overcome the effects of the meridional circulation.

A more detailed look at the eddy-mean flow effects was made using the 3-hourly fields saved during part of the experiment. Figure 27 shows the equatorial zonalmean zonal wind averaged over December of year 28 through January of year 29, and also averaged over the December-January period one year later. The QBO, of course, is almost out of phase between the two periods, with strong westerlies below $10 \mathrm{mb}$ in winter $28 / 29$ and easterlies in winter 29/30. Figure 28 shows an analysis of the vertical eddy flux of zonal momentum, $-\overline{u^{\prime} \omega^{\prime}}$, at the equator during these two periods. The fields have been decomposed into eastward and westward propagating components using the space-time Fourier analysis technique of Hayashi (1971). For each of the westward and eastward components three curves are shown: for all the resolved zonal scales (wavenumbers 1-50), for just wavenumbers $1-15$, and for just wavenumbers 15. The contrast between the two periods is striking, particularly below $10 \mathrm{mb}$. The attenuation of eastward (westward) wave fluxes is greatly enhanced in westerly (easterly) mean wind. This is consistent with the behavior expected from simple models of the dissipation of vertically propagating waves (e.g., Lindzen 1971, 1972). Of course the dropoff in momentum flux seen in Fig. 28 can reflect not only wave dissipation, but also a spreading out of the waves from the equator to higher latitudes [likely to be significant for the gravity waves, see Manzini and Hamilton (1993)]. 

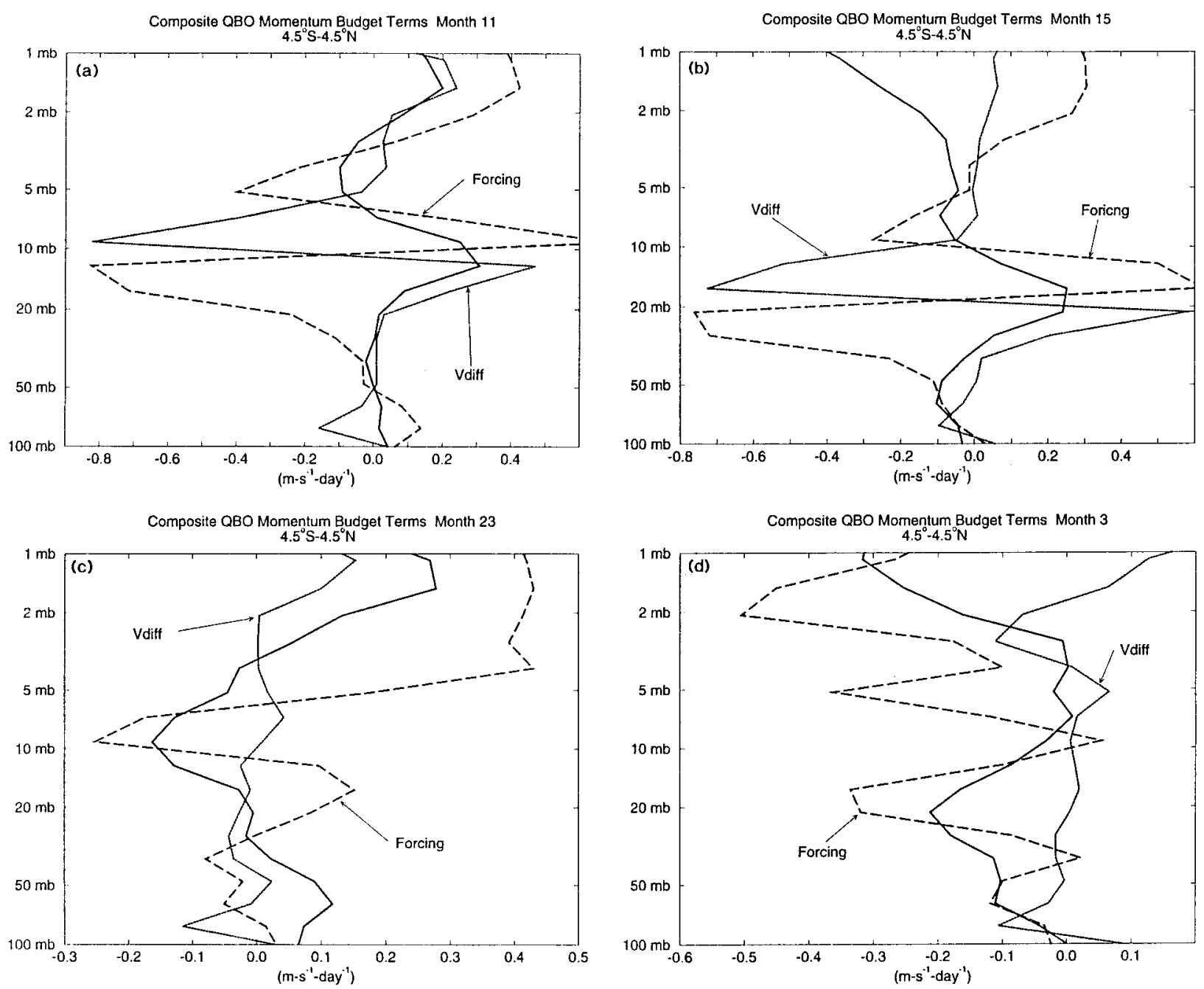

FIG. 26. As in Figs. 24 and 25 but showing some different components of the momentum budget. The long-dashed curve shows the contribution from the imposed QBO relaxation included in this model experiment and dotted curve shows the vertical subgrid-scale diffusion of momentum.

In summary, the model does not produce a QBO spontaneously, but does exhibit some of the behavior that is thought involved in generating the QBO in the real atmosphere. The imposition of a mean jet of one sign in the lower stratosphere results in a filtering of the vertically propagating wave field that produces eddy driving of the mean flow in the opposite sense at higher levels. In simple models this effect can produce oscillating, downward-propagating QBO wind reversals (e.g., Holton and Lindzen 1972). The full GCM is apparently inhibited from oscillating by the vertical advection of mean-flow momentum by the mean meridional circulation. The wave driving of the mean flow is not sufficient to overcome this advective effect and also provide the needed mean flow accelerations. This seems to be a particular problem in the easterly acceleration phase in the lower stratosphere. There is also a complication in the model of a vertical diffusion that be- comes very large when the mean shears approach those observed in the real QBO. This by itself would likely prevent the model from developing a QBO with realistically strong shears, but it is not the basic cause of the problem with the control model (since the control model circulation never develops mean shears strong enough to activate the strong vertical diffusion).

\section{Stationary wave response in the Tropics}

As noted earlier, both observations and the present model simulation show a significant effect of the QBO on the stationary wave pattern in the $\mathrm{NH}$ winter extratropics. Some of the wave activity associated with quasistationary eddies generated in the extratropical region is known to propagate equatorward (e.g., Andrews et al. 1987). The propagation of such waves to very low latitudes (and perhaps even across the equator) is likely 


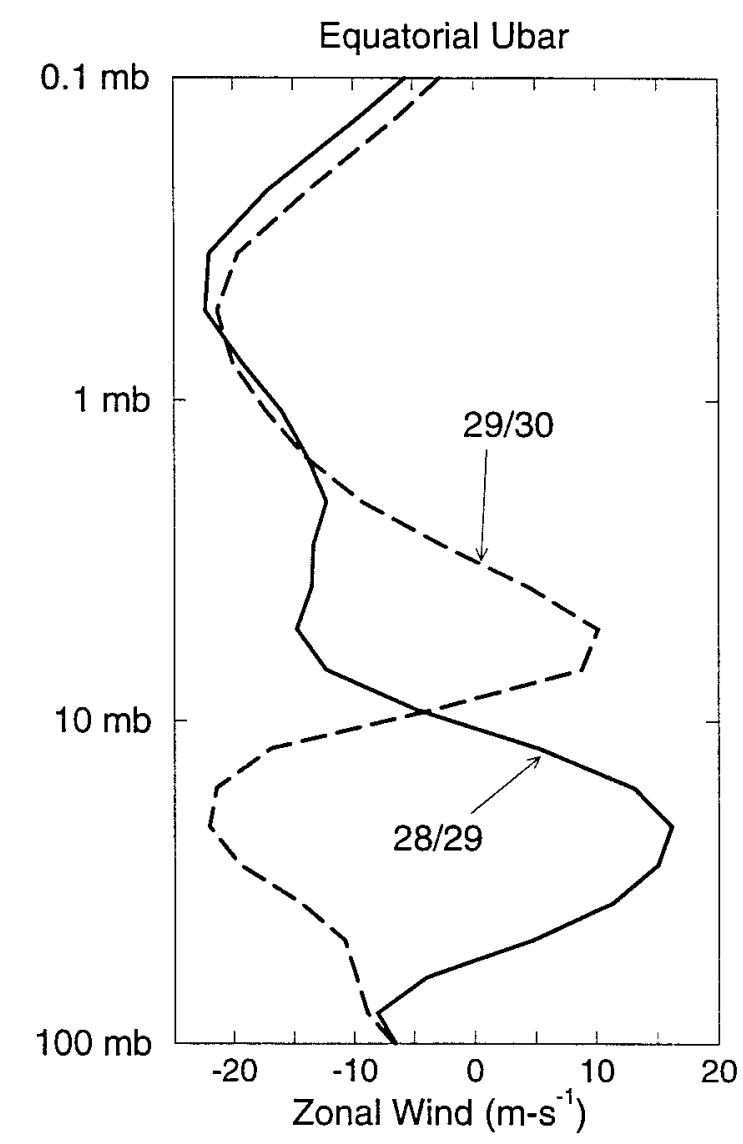

FIG. 27. Zonal-mean zonal wind at the equator averaged over the two month periods: December 28-January 29 (solid), and December 29-January 30 (dashed).

to be strongly affected by the QBO. In the east phase of the QBO such waves will encounter critical surfaces on the poleward flank of the tropical easterlies, whereas in the west QBO phase the mean flow will be westerly throughout the entire winter hemisphere. Observational studies of the stationary wave field at low latitudes is hampered by a scarcity of in situ wind observations, and by the difficulty in using satellite-derived geopotential to determine the wind field near the equator. Recently, the availability of several years of direct satellitedetermined winds from the UARS High Resolution Doppler Imager instrument has enabled some progress to be made (Ortland et al. 1996). Ortland (1997) finds significant deviations from zonal symmetry in the prevailing wind field along the equator when the zonalmean zonal wind is westerly, but not when it is easterly. This is particularly apparent at and above $35 \mathrm{~km}$ in his data. O'Sullivan (1997) has examined the $\mathrm{N}_{2} \mathrm{O}$ fields at midstratospheric levels determined from the UARS Cryogenic Limb Array Etalon Spectrometer instrument, and has found evidence for cross-equatorial radiation of quasi-stationary Rossby waves at levels where the equatorial mean flow is westerly. In this section the behavior
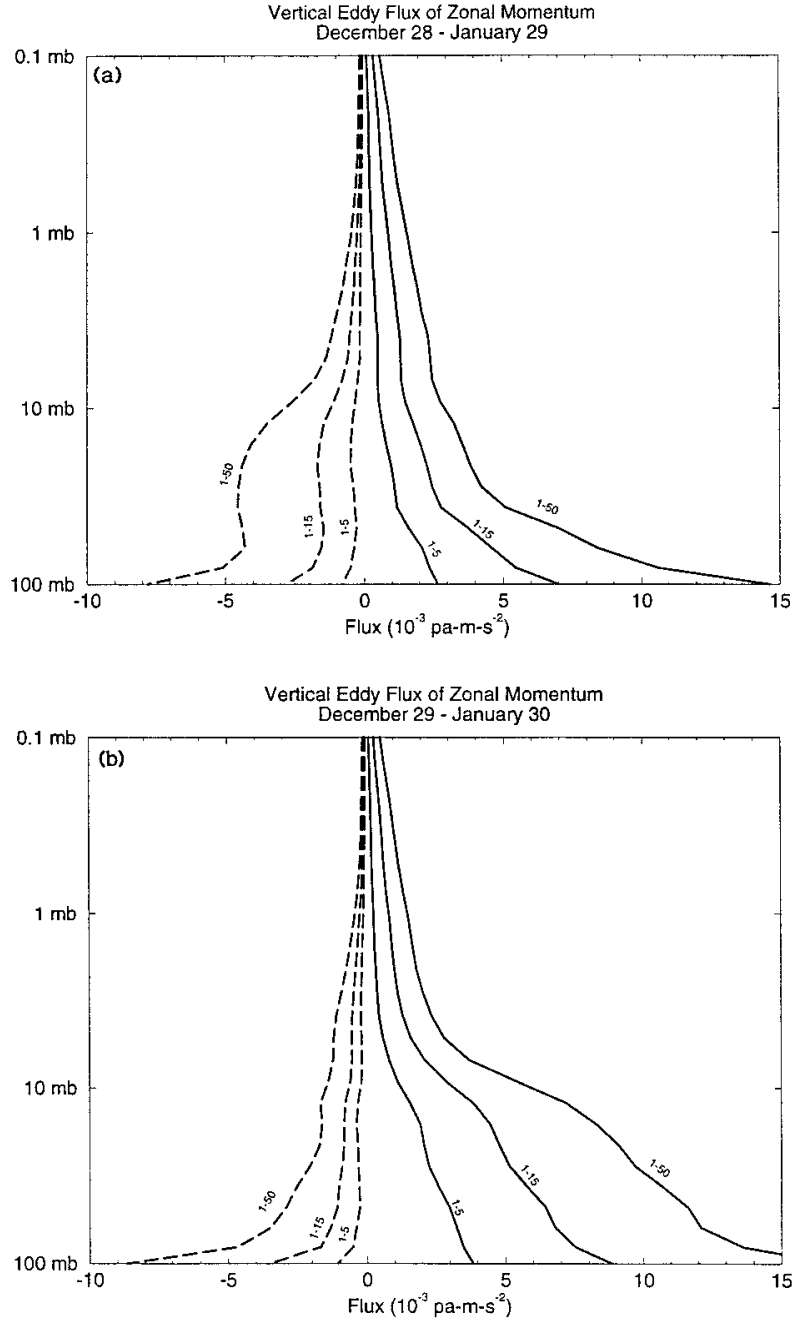

FIG. 28. Vertical flux of zonal momentum $-\overline{u^{\prime} \omega^{\prime}}$ associated with eddies in the model. In each panel the solid (dashed) curves are for waves propagating eastward (westward) relative to the ground. Each curve is labeled according to the number of zonal wavenumbers included in the calculation. The curves labeled 1-50 include all resolved wavenumbers: (a) for the period December year 28 through January year 29, (b) for the period December year 29 through January year 30 .

of the low-latitude stationary waves in the SKYHI model with the imposed zonal-mean QBO will be discussed.

Composites of the DJF meridional wind for east and west phases of the QBO were constructed (not shown). At levels around or above $10 \mathrm{mb}$ these show the $\mathrm{NH}$ stationary wave pattern extending into equatorial latitudes whenever the equatorial mean wind (at the height being considered) is westerly. The zonal contrasts in DJF average meridional wind at the equator are fairly modest $\left(\sim 1 \mathrm{~m} \mathrm{~s}^{-1}\right.$ at most), but the effects on the zonal wind are stronger. Figure 29 shows the DJF mean 9.22$\mathrm{mb}$ equatorial zonal wind plotted for 18 separate years at three latitudes. At the equator (middle panel) the expected spread in zonal-mean values depending on the phase of the QBO is quite apparent. The interesting 

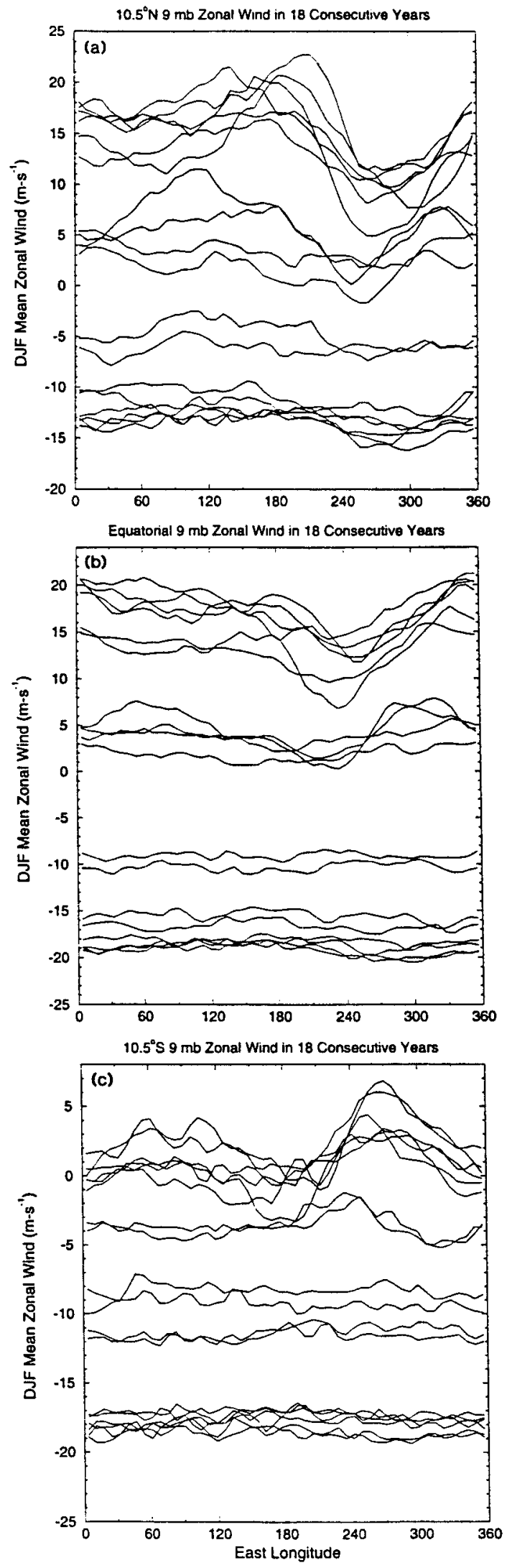

feature here is the significant zonal asymmetries that appear in those winters with westerly zonal-mean winds. In individual winters the contrast along the equator can be as large as $\sim 8 \mathrm{~m} \mathrm{~s}^{-1}$. However, in those winters with easterly mean wind, the zonal variations are very small. The same quantities, but for $10.5^{\circ} \mathrm{N}$ and $10.5^{\circ} \mathrm{S}$ are shown in the other panels of Fig. 29. The eddy component of the zonal wind is larger at $10.5^{\circ} \mathrm{N}$ than at the equator, but it still displays a very strong contrast between seasons with mean westerlies and those seasons with mean easterlies. The stationary eddy field is weaker, but still quite apparent, at $10.5^{\circ} \mathrm{S}$. The pattern is consistent with the notion that stationary waves of extratropical origin propagate into the Tropics from the winter hemisphere, but that their penetration is strongly restricted by the presence of zonal-mean easterlies at low latitudes. It is striking that the penetration of a significant standing wave to the equator appears quite predictably in each individual winter with mean westerlies.

The zonal variations seen in Fig. 29b effectively represent a significant zonal asymmetry in the equatorial zonal wind QBO. As noted in the introduction, the view that the equatorial $\mathrm{QBO}$ is zonally symmetric depends largely on early studies of radiosonde data at and below $50 \mathrm{mb}$. The zonal asymmetries in the DJF mean equatorial winds in the model are strongest near the 9.22$\mathrm{mb}$ level and drop off in magnitude at both higher and lower levels. When the analysis is repeated for the zonal wind at $47.9 \mathrm{mb}$ (not shown), the stationary waves at low latitudes are in fact quite weak. So the present results are consistent with the observational study of Belmont and Dartt (1968). Unfortunately, detailed confirmation of the model results near $10 \mathrm{mb}$ from radiosonde data is difficult, given the limited data now available. The current reliance on Singapore as the only near-equatorial station regularly reporting middle stratospheric winds may be a significant limitation for monitoring the QBO. If the present model results are correct, then the winter-mean Singapore zonal winds at $10 \mathrm{mb}$ may differ from the true zonal-mean values by $\sim 5 \mathrm{~m} \mathrm{~s}^{-1}$ during the westerly QBO phase.

\section{Conclusions}

This paper has described the response of a comprehensive GCM to the imposition of a tropical momentum source designed to force the equatorial stratospheric winds to undergo a 27-month oscillation. The imposed forcing succeeded in driving a realistic QBO near the

FIG. 29. (a) The December-February average zonal wind at $10.5^{\circ} \mathrm{N}$ and $9.22 \mathrm{mb}$ plotted for each of 18 individual years. (b) As in panel (a) but for the grid row at the equator. (c) As in panel (a) but for the $10.5^{\circ} \mathrm{S}$ grid row. Note that the equatorial zonal-mean prevailing wind in observations is often inferred from measurements at Singapore $\left(1.4^{\circ} \mathrm{N}, 103.9^{\circ} \mathrm{E}\right)$ 
equator without producing any obvious discontinuities in the flow between the strongly constrained tropical region and higher latitudes. The observed QBO in tropical zonal-mean temperature is well reproduced in the model, which is an indication that the vertical shears resulting from the QBO forcing are realistic.

The effects of the imposed QBO on the interannual variability of the $\mathrm{NH}$ winter stratospheric circulation were examined in detail. The phase of the tropical QBO was shown to significantly affect the occurrence of sudden warmings as well as the winter-mean of the extratropical circulation. The sign and magnitude of the influence was in quite good agreement with available observations. On average, the high-latitude DJF polar temperatures over a deep stratospheric layer were found to be $\sim 3^{\circ} \mathrm{C}$ warmer during the east than the west QBO phase. The peak zonal-mean zonal wind speeds in the DJF mean polar vortex were also $\sim 10 \mathrm{~m} \mathrm{~s}^{-1}$ weaker during the east QBO phase than during the west phase. While these QBO effects are important, the tropical QBO accounts for only a part of the extratropical variance, and there is nothing approaching a one-to-one relationship between the tropical QBO phase and the state of the winter-mean $\mathrm{NH}$ polar vortex (or the occurrence of sudden warmings). However, including the QBO in the model may improve some biases seen in the control version, notably in introducing a more biennial character to the time series of high-latitude temperatures and in reducing the model overprediction of the interannual variance of high-latitude Decembermean stratospheric temperatures.

The model results show that at times of mean tropical westerlies there should be some significant penetration of stationary waves from the $\mathrm{NH}$ winter midlatitudes to the equatorial region and even into the SH. Contrasts in the seasonal-mean zonal wind along the equator near $10 \mathrm{mb}$ can exceed $8 \mathrm{~m} \mathrm{~s}^{-1}$. If this is true in the real atmosphere as well, then the current radiosonde network must be regarded as somewhat inadequate for monitoring the prevailing winds near the equator.

The only clear tropospheric effects of the imposed QBO were in the high-latitude NH winter. The tendency for a weaker polar vortex during the tropical easterly QBO phase extends down to midtropospheric levels. The EP flux emerging upward from the upper troposphere of the NH winter extratropics was also significantly correlated with the phase of the equatorial QBO. This is an indication of how complicated the full atmospheric response to the tropical QBO can be, and is an effect that might be missing from some more idealized mechanistic models of the middle atmosphere. There were no clear effects of the imposed QBO on the tropical tropospheric circulation or precipitation.

The momentum budget in the equatorial region through the QBO cycle was examined in detail. The vertically propagating waves in the model are very strongly affected by the QBO mean flow changes. In accord with theoretical expectations (e.g., Lindzen
1971, 1972), the eastward (westward) propagating waves are attenuated more strongly in westerly (easterly) mean flow. This behavior is seen in both planetaryscale equatorial waves and the smaller-scale resolved gravity waves. As noted by Holton and Lindzen (1972) this filtering effect can lead to wave driving of the mean flow characterized by descending shear zones of alternating direction, just as in the QBO. In fact the total wave driving of the equatorial mean flow in this model experiment approximates or even exceeds the mean flow accelerations over much of the QBO cycle. In this sense one can say that the model meets at least one necessary condition for self-consistently generating the QBO. However, there are at least three complicating factors that presumably inhibit the spontaneous generation of a realistic QBO. One is the weakness of the wave-induced easterly forcing below about $20 \mathrm{mb}$. Another is the significant effect of the momentum advection by the residual circulation, which acts to oppose the realized mean flow accelerations. Finally, the vertical subgridscale diffusion of zonal momentum is a very large term in the momentum budget, particularly near the peak westerly shears.

Any long-lived chemical trace species included in the model would be expected to respond to the imposed dynamical QBO. The QBO experiment reported here incorporated $\mathrm{N}_{2} \mathrm{O}$ as a prognostic variable, and indeed a QBO in the tropical stratospheric $\mathrm{N}_{2} \mathrm{O}$ concentrations appeared quite clearly in the results (not shown). Another version of the SKYHI model including a rather complete ozone chemistry is now under development. Preliminary results suggest that when the QBO momentum source is included in this model, a quite realistic tropical ozone QBO results (L. Perliski 1997, personal communication). Future efforts will focus on diagnosing the detailed chemical and transport processes involved in the ozone QBO.

Acknowledgments. The author wishes to thank J. D. Mahlman, L. Perliski, R. J. Wilson, and the journal referees for comments on the manuscript. The expression for the vertical structure of the QBO in appendix A was developed in collaboration with E. Manzini.

\section{APPENDIX}

\section{Time-Height Structure of the Imposed Relaxation}

The time-height dependence of the QBO wind distribution described in section 2 is given by

$$
U\left(p_{k}, t\right)=F_{k} \sum_{n=1}^{6} A_{n} \sin \left[2 \pi n \frac{\left(t+Z_{k}-50\right)}{27}\right],
$$

where $p_{k}$ is the pressure of the $k$ th level from the top, $Z_{k}$ is a standard height (in $\mathrm{km}$ ) above sea level for level $k$, and $t$ is the time in months. The amplitudes $A_{n}$ are $1.0,0.15,0.20,0.08,0.04$ and 0.02 for $n=1,2,3,4$, 
TABle A1. Parameters for Formula A1.

\begin{tabular}{cccc}
\hline \hline $\begin{array}{c}\text { Level } \\
\text { number, } k\end{array}$ & $\begin{array}{c}\text { Pressure } \\
(\mathrm{mb})\end{array}$ & $\begin{array}{c}\text { Standard height } \\
(\mathrm{km})\end{array}$ & $\begin{array}{c}F_{\mathrm{k}} \\
\left(\mathrm{m} \mathrm{s}^{-1}\right)\end{array}$ \\
\hline 11 & 2.07 & 42.84 & 1.00 \\
12 & 2.82 & 40.47 & 3.82 \\
13 & 3.83 & 38.29 & 7.73 \\
14 & 5.16 & 36.01 & 12.68 \\
15 & 6.92 & 33.89 & 17.57 \\
16 & 9.22 & 31.85 & 22.02 \\
17 & 12.2 & 29.88 & 25 \\
18 & 16.2 & 27.96 & 25 \\
19 & 21.3 & 26.09 & 25 \\
20 & 28.0 & 24.28 & 22.31 \\
21 & 36.7 & 22.51 & 16.49 \\
22 & 47.9 & 20.79 & 10.59 \\
23 & 62.4 & 19.12 & 5.52 \\
24 & 80.7 & 17.49 & 1.93 \\
25 & 103 & 15.90 & 0.2 \\
\hline
\end{tabular}

5 , and 6 , respectively. The overall amplitude modulations, $F_{k}$, and standard heights are given in Table A1.

\section{REFERENCES}

Andrews, D. G., J. D. Mahlman, and R. W. Sinclair, 1983: EliassenPalm flux diagnostics of wave-mean flow interaction in the GFDL "SKYHI" general circulation model. J. Atmos. Sci., 40, 2768-2784.

_ J. R. Holton, and C. B. Leovy, 1987: Middle Atmosphere Dynamics. Academic Press, 489 pp.

Austin, J., and N. Butchart, 1994: The influence of climate change and the timing of stratospheric warmings on Arctic ozone depletion. J. Geophys. Res., 99, 1127-1145.

- - , and K. P. Shine, 1992: Possibility of an Arctic ozone hole in a doubled- $\mathrm{CO}_{2}$ climate. Nature, 360, 221-225.

Balachandran, N. K., and D. Rind, 1995: Modeling the effects of solar variability and the $\mathrm{QBO}$ on the troposphere/stratosphere system. Part I: The middle atmosphere. J. Climate, 8, 20582079.

— A. Plumb, R. Suozzo, and D. Rind, 1991: The QBO and stratospheric warming-model results. J. Geomagn. Geoelectr., 43 (Suppl.), 741-757.

Baldwin, M. P., and T. J. Dunkerton, 1991: Quasi-biennial oscillation above 10 mb. Geophys. Res. Lett., 18, 1205-1208.

Belmont, A. D., and D. G. Dartt, 1968: Variation with longitude of the quasi-biennial oscillation. Mon. Wea. Rev., 96, 767-777.

,-- , and G. D. Nastrom, 1974: Periodic variations in stratospheric zonal wind from 20 to $65 \mathrm{~km}$ at $80^{\circ} \mathrm{N}$ to $70^{\circ} \mathrm{S}$. Quart. J. Roy. Meteor. Soc., 100, 203-211.

Bowman, K. P., 1989: Global patterns of the quasi-biennial oscillation in total ozone. J. Atmos. Sci., 46, 3328-3343.

Burrage, M. D., R. A. Vincent, H. G. Mayr, W. R. Skinner, N. F. Arnold, and P. B. Hays, 1996: Long term variability in the equatorial mesosphere and lower thermosphere zonal winds. J. Geophys. Res., 101, 12 847-12 854

Chen, P., 1996: The influences of zonal flow on wave breaking and tropical-extratropical interaction in the lower stratosphere. J. Atmos. Sci., 53, 2379-2392.

Dunkerton, T. J., and D. P. Delisi, 1985: Climatology of the equatorial lower stratosphere. J. Atmos. Sci., 42, 1199-1208.

_ etary wave fluxes in the Northern Hemisphere winter. J. Atmos. Sci., 48, 1043-1061.

- D. P. Delisi, and M. P. Baldwin, 1988: Distribution of major stratospheric sudden warmings in relation to the quasi-biennial oscillation. Geophys. Res. Lett., 15, 136-139.
Ebdon, R. A., 1975: The quasi-biennial oscillation and its association with tropospheric circulation patterns. Meteor. Mag., 104, 282297.

Garcia, R. R., and S. Solomon, 1987: A possible relationship between interannual variability in Antarctic ozone and the quasi-biennial oscillation. Geophys. Res. Lett., 14, 848-851.

_ , and B. A. Boville, 1994: "Downward control" of the mean meridional circulation and temperature distribution of the polar winter stratosphere. J. Atmos. Sci., 51, 2238-2245.

- T. J. Dunkerton, R. S. Lieberman, and R. A. Vincent, 1997: Climatology of the semiannual oscillation of the tropical middle atmosphere. J. Geophys. Res., 102, 26019-26032.

Geller, M. A., W. Shen, M. Zhang, and W.-W. Tan. 1997: Calculations of the stratospheric QBO for time-varying forcing. J. Atmos. Sci., 54, 883-894.

Gray, W. M., 1984: Atlantic hurricane frequency. Part I: El Niño and $30 \mathrm{mb}$ quasi-biennial oscillation influences. Mon. Wea. Rev., 112, 1649-1668.

—, J. D. Schaeffer, and J. A. Knaff, 1992a: Hypothesized mechanism for stratospheric QBO influence on ENSO variability. Geophys. Res. Lett., 19, 107-110.

_ C. W. Landsea, P. W. Mielke Jr., and K. J. Berry, 1992b: Predicting Atlantic seasonal hurricane activity 6-11 months in advance. Wea. Forecasting, 7, 440-455.

Hamilton, K., 1984: Mean wind evolution through the quasi-biennial cycle in the tropical lower stratosphere. J. Atmos. Sci., 41, 21132125.

- 1985: The initial westerly acceleration phase of the stratospheric quasi-biennial oscillation as revealed in FGGE analyses. Atmos.-Ocean, 23, 188-192.

_- 1989: Interhemispheric asymmetry and annual synchronization of the ozone quasi-biennial oscillation. J. Atmos. Sci., 46, 10191025 .

_ $1995 \mathrm{a}$ : Interannual variability in the Northern Hemisphere winter middle atmosphere in control and perturbed experiments with the SKYHI general circulation model. J. Atmos. Sci., 52, 4466.

_ 1995b: Comprehensive modelling of the middle atmospheric climate: Some recent results. Climate Dyn., 11, 223-241.

— 1995c: Comment on "Global QBO in circulation and ozone. Part I: Reexamination of observational evidence." J. Atmos. Sci., 52, 1834-1838.

- and L. Yuan, 1992: Experiments on tropical stratospheric mean wind variations in a spectral general circulation model. J. Atmos. Sci., 49, 2464-2483.

, R. J. Wilson, J. D. Mahlman, and L. J. Umscheid, 1995: Climatology of the GFDL SKYHI troposphere-stratosphere-mesosphere general circulation model. J. Atmos. Sci., 52, 5-43.

Hasebe, F., 1983: Interannual variations of total ozone revealed from Nimbus-4 BUV and ground-based observations. J. Geophys. Res., 88, 6819-6834.

Hayashi, Y., 1971: A generalized method of resolving disturbances into progressive and retrogressive by space Fourier and time cross-spectral analysis. J. Meteor. Soc. Japan, 49, 125-128.

Haynes., P. H., C. J. Marks, M. E. McIntyre, T. G. Shepherd, and K. P. Shine, 1991: On the "downward control" of extratropical diabatic circulations by eddy-induced mean zonal forces. J. Atmos. Sci., 48, 651-678.

Hess, P. G., and D. O'Sullivan, 1995: A three-dimensional modelling study of the extratropical quasi-biennial oscillation in ozone. $J$. Atmos. Sci., 52, 1539-1554.

Holton, J. R., 1989: Influence of the annual cycle in the meridional transport on the quasi-biennial oscillation in total ozone. J. Atmos. Sci., 46, 1434-1439.

— biennial cycle of the tropical stratosphere. J. Atmos. Sci., 29, 1076-1080.

, and H.-C. Tan, 1980: The influence of the equatorial quasibiennial oscillation on the global circulation at $50 \mathrm{mb}$. J. Atmos. Sci., 40, 1410-1425. 
- and -1982 : The quasi-biennial oscillation in the Northern Hemisphere lower stratosphere. J. Meteor. Soc. Japan, 60, 140147.

- , and J. Austin, 1991: The influence of the QBO on sudden stratospheric warmings. J. Atmos. Sci., 48, 607-618.

— , P. H. Haynes, M. E. McIntyre, A. R. Douglass, R. B. Rood, and L. Pfister, 1995: Stratosphere-troposphere exchange. Rev. Geophys., 33, 403-439.

Labitzke, K., 1982: On the interannual variability of the middle stratosphere during northern winters. J. Meteor. Soc. Japan, 60, 124139

Levy, H., J. D. Mahlman, and W. J. Moxim, 1982: Tropospheric $\mathrm{N}_{2} \mathrm{O}$ variability. J. Geophys. Res., 87, 3061-3080.

Lindzen, R. S., 1971: Equatorial planetary waves in shear: Part I. $J$. Atmos. Sci., 28, 609-622.

- 1972: Equatorial planetary waves in shear: Part II. J. Atmos. Sci., 29, 1452-1463.

— lation. J. Atmos. Sci., 25, 1095-1107.

Manzini, E., and K. Hamilton, 1993: Middle atmospheric traveling waves forced by latent and convective heating. J. Atmos. Sci., 50, 2180-2200.

— from a general circulation model and observations. Climate Dyn., 12, 615-639.

Maruyama, T., and Y. Tsuneoka, 1988: Anomalously short duration of easterly wind phase of the QBO at $50 \mathrm{hPa}$ in 1987 and its relationship to an El Niño event. J. Meteor. Soc. Japan, 66, 629633.

Mukherjee, B. K., K. Indira, R. S. Reddy, and B. V. Ramana Murty, 1985: Quasi-biennial oscillation in stratospheric zonal wind and Indian summer monsoon. Mon. Wea. Rev., 113, 1421-1424.

Naujokat, B., 1986: An update of the observed quasi-biennial oscillation of the stratospheric winds over the tropics. J. Atmos. Sci., 43, 1873-1877.

Oltmanns, S. J., and J. London, 1982: The quasi-biennial oscillation in atmospheric ozone. J. Geophys. Res., 87, 8981-8989.

Ortland, D. A., 1997: Rossby wave propagation into the tropical stratosphere observed by the High Resolution Doppler Imager. Geophys. Res. Lett., 24, 1999-2002.

- W. R. Skinner., P. B. Hays, M. D. Burrage, R. S. Lieberman, A. R. Marshall, and D. A. Gell, 1996: Measurements of stratospheric winds by the high resolution Doppler imager. J. Geophys. Res., 101, $10351-10363$.

O’Sullivan, D., 1997: Cross-equatorially radiating stratospheric Rossby waves. Geophys. Res. Lett., 24, 1483-1486.

- , and M. L. Salby, 1990: Coupling of the quasi-biennial oscillation and the extratropical circulation in the stratosphere through planetary wave transport. J. Atmos. Sci., 47, 650-673.

— tion's effect on the winter stratospheric circulation. J. Atmos. Sci., 49, 2437-2448.

— , and T. J. Dunkerton, 1994: Seasonal development of the extratropical QBO in a numerical model of the middle atmosphere. J. Atmos. Sci., 51, 3706-3721.

—, and P. Chen, 1996: Modeling the quasi-biennial oscillation's influence on isentropic tracer transport in the subtropics. J. Geophys. Res., 101, 6811-6821.

Pawson, S., and M. Fiorino, 1996: Structure of the tropical lower stratosphere as revealed by three reanalysis data sets. Program for Climate Model Diagnosis and Intercomparison Report 35, Lawrence Livermore Laboratory, 35 pp. [Available from Program for Climate Model Diagnosis and Intercomparison, Lawrence Livermore Laboratory, Livermore, CA 94550.]

Plumb, R. A., 1982: Zonally symmetric Hough modes and meridional circulations in the middle atmosphere. J. Atmos. Sci., 39, 983991.

Polvani, L. M., D. W. Waugh, and R. A. Plumb, 1995: On the subtropical edge of the stratospheric surf zone. J. Atmos. Sci., 52, 1288-1309.

Randel, W. J., 1992: Global atmospheric circulation statistics, 1000$1 \mathrm{mb}$. NCAR Tech. Note TN-366+STR, $256 \mathrm{pp}$. [Available from the National Center for Atmospheric Research, Boulder, CO 80307.]

_ , and F. Wu, 1996: Isolation of the ozone QBO in SAGE II data by singular-value decomposition. J. Atmos. Sci., 53, 2546-2559.

Reed, R. J., 1964: A climatology of wind and temperature in the tropical stratosphere between $100 \mathrm{mb}$ and $10 \mathrm{mb}$. Rep. 26-0564092, U.S. Navy Research Facility, Monterey, CA, 56 pp. [Available from U.S. Naval Research Laboratory, Monterey, CA 93943.]

Swinbank, R., and A. O'Neill, 1994: A stratosphere-troposphere data assimilation system. Mon. Wea. Rev., 122, 686-702.

Takahashi, M., 1996: Simulation of the stratospheric quasi-biennial oscillation using a general circulation model. Geophys. Res. Lett., 23, 661-664.

Trenberth, K., 1980: Atmospheric quasi-biennial oscillations. Mon. Wea. Rev., 108, 1370-1377.

Tung, K. K., and H. Yang, 1994: Global QBO in circulation and ozone. Part I: Reexamination of observational evidence. J. Atmos. Sci., 51, 2699-2707.

Veryard, R. G., and R. A. Ebdon, 1961: Fluctuations in tropical stratospheric winds. Meteor. Mag., 90, 127-143.

Yulaeva, E., J. R. Holton, and J. M. Wallace, 1994: On the cause of the annual cycle in tropical lower stratospheric temperature. $J$. Atmos. Sci., 51, 169-174.

Zawodny, J. M., and M. P. McCormick, 1991: Stratospheric aerosol and gas experiment II measurements of the quasi-biennial oscillations in ozone and nitrogen dioxide. J. Geophys. Res., 96, 9371-9377. 